\title{
CFD simulation of near-field pollutant dispersion in the urban environment: A review of current modeling techniques
}

\author{
Yoshihide Tominaga a and Ted Stathopoulos ${ }^{\mathrm{b}}$ \\ ${ }^{a}$ Department of Architecture and Building Engineering, Niigata Institute of Technology, Kashiwazaki, Niigata, \\ Japan,tominaga@abe.niit.ac.jp \\ ${ }^{b}$ Centre for Building Studies, Department of Building, Civil and Envir. Engineering, Concordia University, Montreal, \\ Quebec, Canada, statho@bcee.concordia.ca \\ *Corresponding author: E-mail addresses: tominaga@abe.niit.ac.jp(Y.Tominaga), statho@bcee.concordia.ca (T. \\ Stathopoulos).
}

\begin{abstract}
Near-field pollutant dispersion in the urban environment involves the interaction of a plume and the flow field perturbed by building obstacles. In the past two decades, micro-scale Computational Fluid Dynamics (CFD) simulation of pollutant dispersion around buildings and in urban areas has been widely used, sometimes in lieu of wind tunnel testing. This paper reviews current modeling techniques in CFD simulation of near-field pollutant dispersion in urban environments and discusses the findings to give insight into future applications. Key features of near-field pollutant dispersion around buildings from previous studies, i.e., three-dimensionality of mean flow, unsteadiness of large-scale flow structure, and anisotropy of turbulent scalar fluxes, are identified and discussed. This review highlights that it is important to choose appropriate numerical models and boundary conditions by understanding their inherent strengths and limitations. Furthermore, the importance of model evaluation was emphasized. Because pollutant concentrations around buildings can vary by orders of magnitudes in time and space, the model evaluation should be performed carefully, while paying attention to their uncertainty. Although CFD has significant potential, it is important to understand the underlying theory and limitations of a model in order to appropriately investigate the dispersion phenomena in question.
\end{abstract}

\section{Keywords}

CFD simulation; near-field; pollutant dispersion; urban environment; modeling technique

Introduction

Air pollution near and around buildings is an important environmental problem. However, it is difficult to predict pollutant dispersion with certainty due to the complex interaction between atmospheric flow and flow around buildings. The pollutants that are brought into the atmosphere by various sources are dispersed (advected and diffused) over a wide range of horizontal length scales, which can be classified into near-field and far-field phenomena. The near-field pollutant dispersion involves the interaction of the plume and the flow field, which may be perturbed by building obstacles. Therefore, it is important to consider when assessing both outdoor and indoor air qualities, because it covers both pollutant concentrations in the surrounding streets and those on building surfaces. For this purpose, the dispersion region that is to be treated is the very short range (i.e. vicinity of the emitting building; within a few hundred meters of the source) rather than the entire (neighbourhood) region of significant impact. Alternatively, in far field phenomena, the horizontal motion prevails over the vertical motion and the influence of individual buildings on a dispersion field becomes relatively small. This issue is discussed mainly in regards to public health regulations on air quality in urban environments.

Until recently, modeling studies on urban air quality were typically conducted by operational models derived from an integral nature of atmospheric dispersion (Stern, 1976; Pasquill and Smith, 1983). The operational models, which are mostly based on the Gaussian dispersion model, are often referred to as 'fast response models'. These 
models are frequently modified for various purposes and have been used for many comprehensive formal evaluations, because they are designed to enable many different cases to be calculated expeditiously (Hanna et al., 2001; Hall et al., 2002). Furthermore, they include many complicated dispersion processes; e.g. atmospheric stratification, buoyancy, chemistry, deposition, concentration fluctuations etc. These are no longer simple Gaussian plume models and are not constrained to simple, straight mean streamlines. For example, ADMS (UK-ADMS), the UK atmospheric dispersion modeling system (Carruthers et al., 1994, 1999; CERC, 2006), is one of the advanced operational models and can take into account a building effect based on a two-plume approach using wake averaged flow values to calculate plume spread (Robins et al., 1997). However, it cannot treat the detailed plume behavior affected by building obstacles explicitly due to the modeling derivation. Therefore, when these models are applied to near-field dispersion in the urban environment, it is important to understand the fundamental concepts used in these models, as suggested by Macdonald (2003).

A method that is more oriented to practical design related to near-field dispersion around a building is based on the so-called ASHRAE model (ASHRAE 2007, 2011). This model was derived from the amassed results of wind tunnel experiments (Wilson, 1982; Wilson, 1983; Wilson and Lamb, 1994) and is also a semi-empirical model. It can be used for determining the appropriate stack height and air intake position for the case of an isolated building. This model is specialized for use in building design, but has limited applicability and less accuracy concerning building configuration details (Hajra et al. 2010; 2011).

In the past two decades, micro-scale Computational Fluid Dynamics (CFD) simulation has been widely used as an emerging analysis method for pollutant dispersion around buildings and in urban areas, sometimes in lieu of wind tunnel testing. The CFD simulation method consists of solving the transport (advection and diffusion) equation of concentration based on the velocity field obtained from the Navier-Stokes equations. CFD can provide detailed information about the relevant flow and concentration variables throughout the calculation domain; however, it is more time-consuming than the two previously mentioned methods. Moreover, it is difficult to implement various dispersion processes such as atmospheric stratification, buoyancy, chemistry etc. to the model, whereas they are easily implemented to the operational models.

Recently, CFD has been studied extensively for the assessment of pollutant dispersion around buildings. However, these studies have been performed with different research purposes, configurations, boundary conditions, and modeling approaches. This makes it difficult to evaluation of the strengths and limitations of CFD for the evaluation of near-field pollutant dispersion in the urban environment. As already mentioned, the near-field dispersion around buildings is characterized by the complex interaction between the atmospheric flow and the flow around buildings. The phenomenon has both meteorological and building aerodynamic aspects, however the majority of previous research has been conducted within the frameworks of each aspect. Hence, a comprehensive review with cross-cutting aspects is required for CFD simulations of near-field pollutant dispersion in the urban area.

Furthermore, for evaluating the quality of CFD simulations, it is necessary to analyze its sensitivity and uncertainty appropriately. ERCOFTAC Best practice guidelines (Casey \& Wintergerste, 2000) gives the best practical advice for achieving high-quality industrial CFD simulations and provides relevant information on the most important issues relevant to the credibility, especially with regard to the most common sources of errors and uncertainties in CFD. Recently, several best practice guidelines have been proposed as verification and validation process of CFD for urban wind environment applications, mainly intended for the prediction of pedestrian level winds (Franke et al., 2004, 2007; Tominaga et al., 2008b). Although these practical guidelines are quite effective in the pollutant dispersion problem, there are additional recommendations specific to the dispersion problem, i.e. requirements of modeling for a contaminant transport equation and sensitivity to wind and other climatic conditions. Robins et al. (2000) investigated uncertainty in CFD predictions of building affected dispersion through the Evaluation of Modeling Uncertainty (EMU) project which involved a group of four organizations undertaking CFD simulations for a series of realistic near-field dispersion test cases (Hall, 1997). The study identified some important qualitative guidelines for good modeling practice to indicate where attention should be focused. In the context of rapid increase of CFD applications to near-field pollutant dispersion around buildings in recent years, it is critical to examine past studies on this area.

This paper reviews the current modeling techniques in CFD simulation of near-field pollutant dispersion in the urban environment and discusses the findings to give insights into future directions of practical applications. In Section 2, previous studies of near-field pollutant dispersion around buildings using CFD are overviewed. The configurations used in previous studies are categorized into four typical cases: an isolated building, a single street canyon, building arrays, and building complexes. Section 3 identifies key features of near-field pollutant dispersion around buildings from previous studies and discusses their relevance in CFD modeling. The importance of proper choice of turbulence models and boundary conditions are emphasized in Sections 4 and 5. Finally, model evaluation methods are discussed, and future directions of practical applications are suggested. 


\subsection{Dispersion around an isolated building}

In order to investigate the basic structure of pollutant dispersion around a building, many research studies examining dispersion around a single obstacle have been conducted by field measurements and wind tunnel experiments (Robins and Castro 1977b; Huber and Snyder, 1982; Ogawa et al., 1983; Li and Meroney, 1983a, b). Flow and contamination patterns around a rectangular building with a rooftop vent are illustrated schematically in Fig. 1 (ASHRAE, 2011). The effluent from the vent is entrained into the zone of recirculating flow behind the downwind face and is brought back up to the roof. The near-field pollutant dispersion is characterized by the interaction between atmospheric flow and flow around a building as expressed in this figure.

Since the 1990s, many numerical studies using CFD have been conducted to investigate the applicability of these models for pollutant dispersion around a single building by the Reynolds-Averaged Navier-Stokes (RANS) approach (Murakami et al., 1990; Zhang et al., 1993; Delaunay et al. 1997; Cowan et al. 1997; Selvam 1997; Li and Stathopoulos, 1997, 1998; Leitl et al., 1997; Meroney et al., 1999) and by Large Eddy Simulation (LES) (Tominaga et al., 1997; Sada and Sato, 2002). The calculations utilized in these studies were conducted with relatively coarse meshes in comparison to present work because of restrictions of computational resources at that time. Moreover, good practice guidelines of CFD for predicting flow fields around buildings have not been established at the time. Therefore, validation of the models had been rather qualitative. Recently, several researchers conducted numerical studies on pollutant dispersion around a generic building model in order to examine CFD modeling performance using established good practice guidelines (Blocken et al., 2008; Tominaga and Stathopoulos, 2009, 2010; Gousseau et al., 2011a). Blocken et al. (2008) showed the semi-empirical ASHRAE model works well in predicting dilution on the roof, but significantly overestimates it on the leeward face in comparison with experimental and CFD simulations. Blocken et al. (2011) provided an overview of recent studies on CFD for dispersion around an isolated cubic building, which was experimentally investigated by Li and Meroney (1983a). This review concluded that the accuracy of pollutant dispersion modeling depends highly on the choice of computational parameters made by the user; also steady RANS simulations provide much lower lateral turbulent diffusion compared with wind tunnel testing. More recently, analyses were performed in order to investigate the mechanism of pollutant diffusion around buildings using the extensive information obtained from LES (Gousseau et al., 2011b, 2012) and Direct Numerical Simulation (DNS) (Rossi et al., 2010). They found that the counter-gradient mechanism that governs turbulent mass transfer is observed in the streamwise direction, i.e. negative turbulent flux is generated in spite of the decrease of the concentration along the plume centerline. In contrast, the distributions of turbulent fluxes support the validity of the gradient-diffusion hypothesis in the vertical and lateral directions. Fig. 2 shows the snapshots of iso-surfaces of scalar concentration from a source on top of the cube at several normalized time intervals from DNS results with $\mathrm{Re}=5,000$ (Rossi et al., 2010). It is observed that coherent structures periodically released from the shear-layer in the symmetry plane result in large-scale scalar blobs.

Among the studies on dispersion around a generic building model, it is important to investigate not only the concentration field but also the relationship between dispersion and flow structure, particularly because most studies focused only on concentration distributions. Therefore, in order to validate and evaluate CFD performance on such phenomena, model results should be compared with experimental data on both the concentration and velocity fields. Robins and Castro (1977b) measured concentration field downstream in the vicinity of a surface mounted cube with a source in a simulated atmospheric boundary layer. Their results were discussed by considering the influence of the flow field in the vicinity of the cube measured in a series of experiments (Robins and Castro, 1977a). Recently, Yoshie et al. (2011) examined flow and dispersion fields with gas emission from the wake region of a single building by detailed experiment, in which instantaneous wind velocity, temperature, and concentration, enabling measurement of turbulent heat flux and turbulent concentration flux, measured simultaneously. 


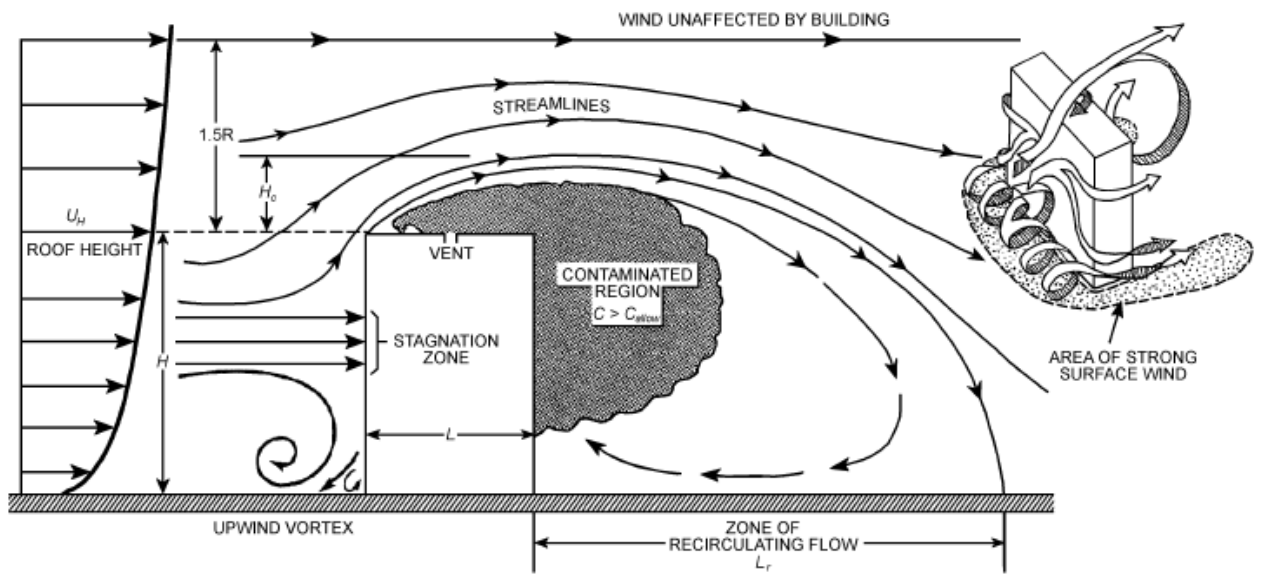

Fig. 1. Schematic diagram of flow and contamination patterns around a rectangular building (ASHRAE 2011).
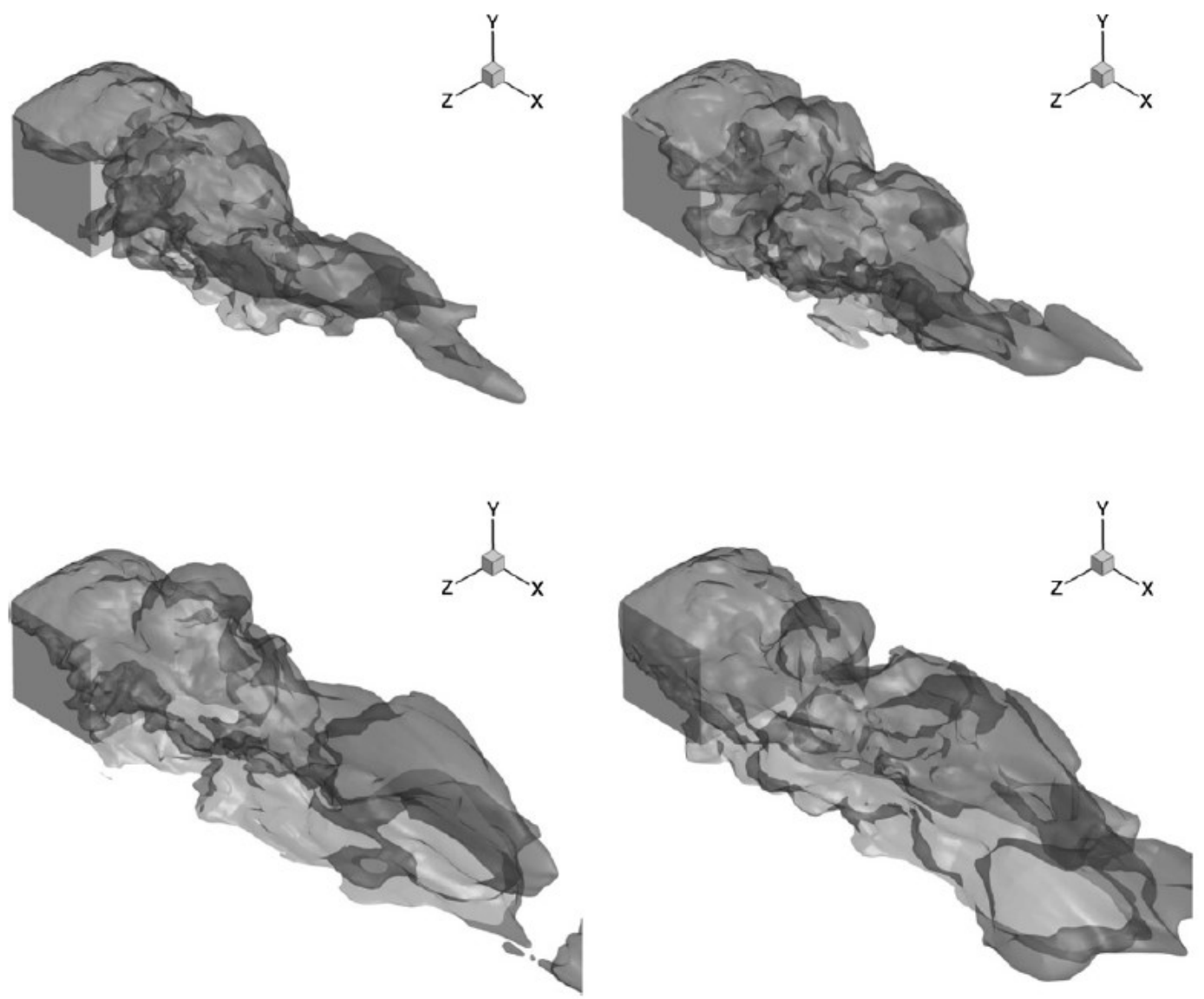

Fig. 2. Iso-surfaces of instantaneous scalar concentration $\mathrm{C} / \mathrm{Cm}=10^{-3}$ from the DNS run with $\mathrm{Re}=5,000$ : upper left panel, $\mathrm{t}^{*}=8$, upper right panel, $\mathrm{t}^{*}=11$, lower left panel, $\mathrm{t}^{*}=18$, lower right panel, $\mathrm{t}^{*}=24$ (Rossi et al., 2010). 
Street canyons are typical architectural structures in urban environments, and they represent highly polluted zones around buildings. In order to minimize the effect of these pollutants in built-up environments, it is necessary to model and accurately predict contaminant dispersion properties in street canyons. Many fundamental studies have been conducted over the years by experimentally modeling the flow and pollutant dispersion in street canyons using wind tunnels. Vardoulakis et al. (2003) and Ahmad et al. (2005) reviewed the measurements and modeling techniques for wind and pollutant transport within street canyons. Recent development of CFD in the study of wind fields and pollutant transport in urban street canyons were reviewed by Li et al. (2006). A considerable amount of research has investigated both the influence of pollutant transport modeling and the influence of inflow conditions, canyon configurations, and other modeling considerations with two-dimensional (2-D) street canyons using RANS (Meroney et al., 1996; Baik and Kim, 1999; Chan et al., 2002; Liu and Barth, 2002; Baik and Kim, 2002; Kim and Baik, 2003; Nazridoust and Ahmadi, 2006) and LES (Liu et al., 2005; Li et al., 2008; Cai et al., 2008; Li et al., 2009; Zhang et al., 2011; Cheng and Liu, 2011a,b). Moreover, significant research has been conducted for generic three-dimensional (3-D) street canyon model using various turbulence models (Hunter et al., 1992; Leitl and Meroney, 1997; Chang and Meroney, 2001; Walton et al., 2002; Walton and Cheng, 2002; Chang and Meroney, 2003; Baik et al., 2003; Yang and Shao, 2008; Murena et al., 2009; Solazzo et al., 2009; Tominaga and Stathopoulos, 2011; Salim et al., 2011; Koutsouaski et al., 2012). A 3-D street canyon, which consists of two or more building blocks, has three determining factors of flow regime, namely the relative height $(\mathrm{H})$, width $(\mathrm{W})$ and length $(\mathrm{L})$ of the canyon, in contrast to a 2-D canyon with two factors, $\mathrm{H}$ and $\mathrm{W}$. Therefore, the flow field formed in a 3-D street canyon is highly complex in comparison to the 2-D case. As summarized in Li et al. (2006), several studies for 3-D street canyons investigated 3-D lateral and secondary flows, which are absent in 2-D simulations, although strongly influence the vertical mixing of pollutant concentration (Hunter et al., 1992; Leitl and Meroney, 1997).

\subsection{Dispersion in and around building arrays (continuous street canyons)}

Many experimental studies have been conducted on pollutant dispersion in and around regularly arrayed obstacles representing urban configurations (Davidson et al., 1995; Macdonald et al., 1998; Yee et al., 2006). It is known that the time-averaged plume profile far enough into obstacle arrays can be well described by a Gaussian distribution when the diffusion parameters are properly constructed (Davidson et al., 1996). By comparing the CFD and the operational models for dispersion over obstacle array configurations, Riddle et al. (2004) observed that CFD is more appropriate for applications that involve complex geometry that cannot be simulated using the ADMS model. However, they also suggested that further studies are required to assess the ability of CFD to calculate dispersion in other situations, such as around groups of buildings and under a range of atmospheric stability conditions. Several parametric studies for model performance on dispersion fields over obstacle arrays using RANS model have carried out (Kim and Baik, 2004; Di Sabatino et al., 2007; Santiago et al., 2007; Martilli and Santiago, 2007; Wang et al., 2009; Buccolieri et al., 2010).

Mock Urban Setting Test (MUST) observation data has been used to validate CFD applied to pollutant diffusion in continuous street canyons (Yee and Biltoit, 2004). A detailed wind tunnel experiment was conducted for this configuration (Harms et al., 2005) and several comparison studies between CFD and experiment were performed (Hanna et al., 2004; Hsieh et al., 2007; Milliez and Carissimo, 2007; Santiago et al., 2010; Dejoan et al., 2010; Antonioni et al., 2012). The data was also used for the model evaluation guidance and protocol document provided by the European COST action 732 (Britter and Schatzmann, 2007; COST Action 732, 2009). The results of the exercise were compiled in several publications (Olesen et al., 2008; Schatzmann et al., 2009; Di Sabatino et al., 2011).

Recently, a number of studies using LES and DNS on dispersion fields over obstacle arrays have been carried out (Shi et al., 2008; Gu et al., 2011; Boppana et al., 2010; Branford et al., 2011). Boppana et al. (2010) demonstrated the influence of the roughness morphology on the dispersion processes and the power of LES for obtaining physically important scalar turbulent flux information. The results of DNS by Branford et al. (2011) largely helped to further understand the processes affecting the plume structure, e.g. channeling, lateral dispersion, detrainment, secondary-source dispersion and plume skewing.

\section{$2.4 \quad$ Dispersion around building complexes}

As the goal of pollutant distribution modeling is to predict pollutant dispersion in an actual urban environment, evaluation studies of dispersion around actual building complexes is critical. In several studies, CFD has been 
applied to pollutant dispersion around actual building groups in urban areas based on RANS (Moon et al., 1997; Hanna et al., 2006; Tseng et al., 2006; Patnaik et al., 2007; Neofytou et al., 2008; Baik et al., 2009; Zhang et al., 2009; Pontiggia et al., 2010, 2011) and LES (Xie and Castro 2009; Xie, 2011; Liu et al., 2011). Although reasonable qualitative outcomes have been obtained from these studies, the results must be evaluated quantitatively. One of the difficulties in CFD studies applied to such building complexes is to obtain reliable experimental and observational results for validation. Recently, detailed wind-tunnel experiments for pollutant dispersion around a building group in downtown Montreal (Stathopoulos et al., 2004) have been used in several studies for CFD validation (Blocken et al., 2008; Lateb et al., 2010; Gousseau et al., 2011a). Furthermore, COST 732 provided the validation protocol for Oklahoma City (OKC) from the Joint Urban 2003 project (JU2003; Allwine et al., 2004). This large urban field program was conducted in Oklahoma City to study atmospheric transport and diffusion in an urban environment. Wind tunnel modeling has also been conducted at the University of Hamburg (Kastner-Klein et al., 2004) to measure high resolution flow and dispersion data sets that supplement JU2003 field data. Several CFD studies have been applied to this subject (Lien et al., 2008). Hanna et al., (2006) compiled computational results of tracer gas dispersion using the urban atmospheric boundary layer scenario in New York City. They found that the five CFD models they compared produce similar wind flow patterns, as well as good agreement with winds observed during a field experiment. Recently, a relevant tracer dispersion experiment conducted in London has been referred to in several CFD simulations (Xie and Castro, 2009; Xie, 2011; Xie et al., 2013). The London experiment formed part of the Engineering and Physical Sciences Research Council (EPSRC), United Kingdom-funded Dispersion of Air Pollution and its Penetration into the Local Environment (DAPPLE) project, which involves field measurements (Arnold et al., 2004; Wood et al., 2009), wind-tunnel experiments (Carpentieri et al., 2009), computational modelling, meteorological measurements, and model development and evaluation.

Key features of near-field pollutant dispersion around buildings

Based on the studies discussed in the preceding section, some features of near-field pollutant dispersion around buildings can be identified by considering bluff body aerodynamics and atmospheric dispersion. The relationship between these features and reminders of the applicability of CFD modeling are explained in this section.

\subsection{Three-dimensionality of mean flow}

Fig. 3 shows main features of flow around cuboids at $0^{\circ}$ and $45^{\circ}$ to the approach flow in a thick boundary layer (Robins and Macdonald, 2001). This figure indicates that the flow structure around a rectangular building is highly three-dimensional. The flow field displayed in Fig. 3(a) is the result of wind impacting normal at a face. Alternatively, when the wind approaches the building on a diagonal, a strong elevated trailing vortex system is generated (Fig. 3(b)). Murakami (1993) schematically demonstrated the distribution of the strain-rate tensors around a simple bluff body as shown in Fig. 4. There exist various sharp velocity gradients in both the streamwise and vertical directions. Since the production of turbulence statistics, such as Reynolds stress, are closely related to the strain-rate tensors, the turbulence characteristics around a bluff body become complex due to the complicated distribution of the strain-rate tensors. Yakhot et al. (2006) also investigated the negative turbulence production was predicted in the region in front of the cube where the main horseshoe vortex originates by DNS computations. Such complex flows should be distinguished from simple boundary layers, for which most RANS models were developed, and are not treated very well in RANS approaches.

Therefore, in the near-field, the pollutant diffusion phenomenon also has a fully three-dimensional nature due to the flow around a building. Many experiments have shown that contaminants can be transported in the opposite direction of the prevailing wind due to the eddies and mean flows induced by buildings (Li and Meroney 1983a, Ogawa et al., 1983). This occurs when the contaminant is caught in sidewall, rooftop, and cavity recirculation zones around isolated buildings. It can be even more prevalent in a group of buildings (e.g., where contaminants can travel upstream in the cavity of one building to the sidewall eddy induced by another building). This behavior makes it very difficult to predict near-field pollutant dispersion around a group of buildings using the semi-empirical approach (e.g., ASHRAE, 2007, 2011). Furthermore, as pointed out by Meroney (1990), the aerodynamic characteristics of a dispersion plume depend upon the shape and intensity of motion within separated flow regions around the obstacle. Belcher (2005) pointed out that the positions of 'shear layers' and 'dividing streamlines' largely control the patterns of mixing and transport in urban areas. He also showed that neighborhood-scale dispersion follows one scaling in the near field and another in the far field after contaminants pass through many intersections. A schematic diagram of plume dispersion through an array of obstacles was demonstrated by Davidson et al. (1995) and is shown in Fig. 5. A number of distinct effects can be observed such as vertical mixing by horseshoe vortices, upwind vertical diffusion, 
smoothing of concentration fluctuations by building wakes, and channeling of plumes along array passages. As a basis for accurate prediction of dispersion using CFD, it is necessary to reproduce such three-dimensional flow structures around buildings.

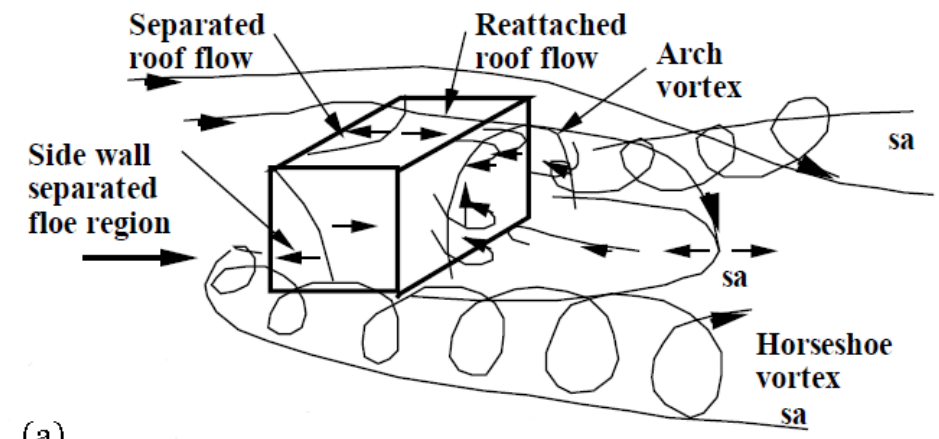

(a)

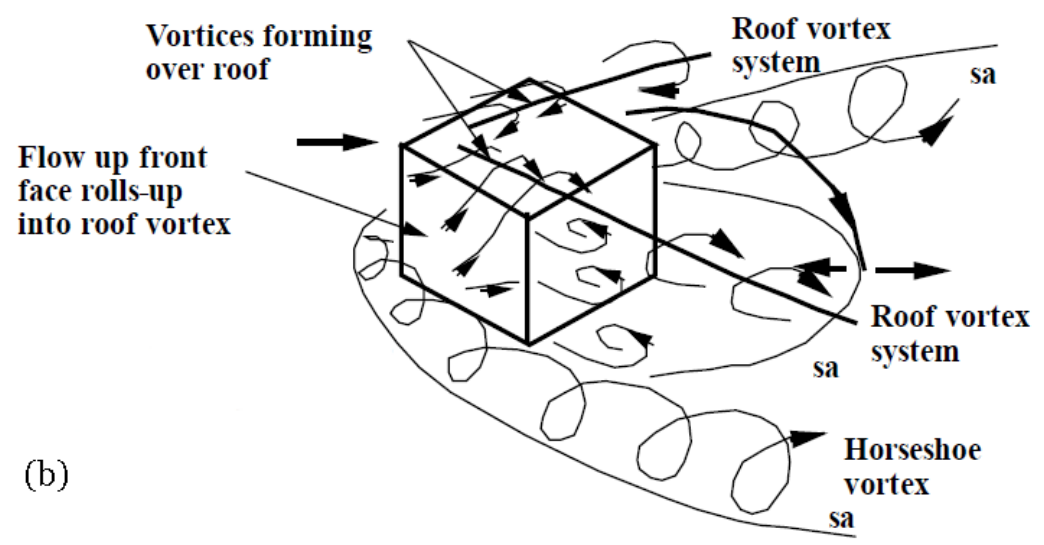

Fig. 3. Main features of flow around cuboids in a thick boundary layer. (a) Mean flow patter around a cuboid normal to the approach flow (lines marked 'sa' denote mean separation/attachment lines). (b) Mean flow pattern around a cuboid at $45^{\circ}$ incidence to the approach flow (Robins and Macdonald, 2001). 

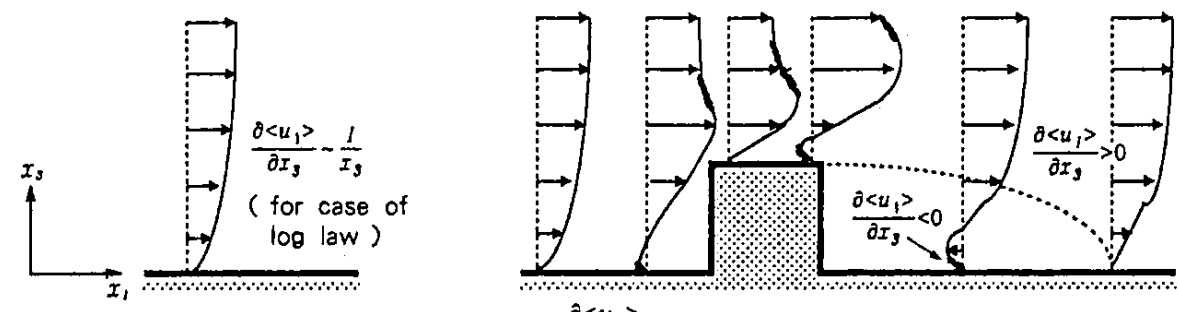

(a) main shear $\frac{\partial\left\langle u_{\rho}\right\rangle}{\partial x_{3}}$

(shadowed lines indicate
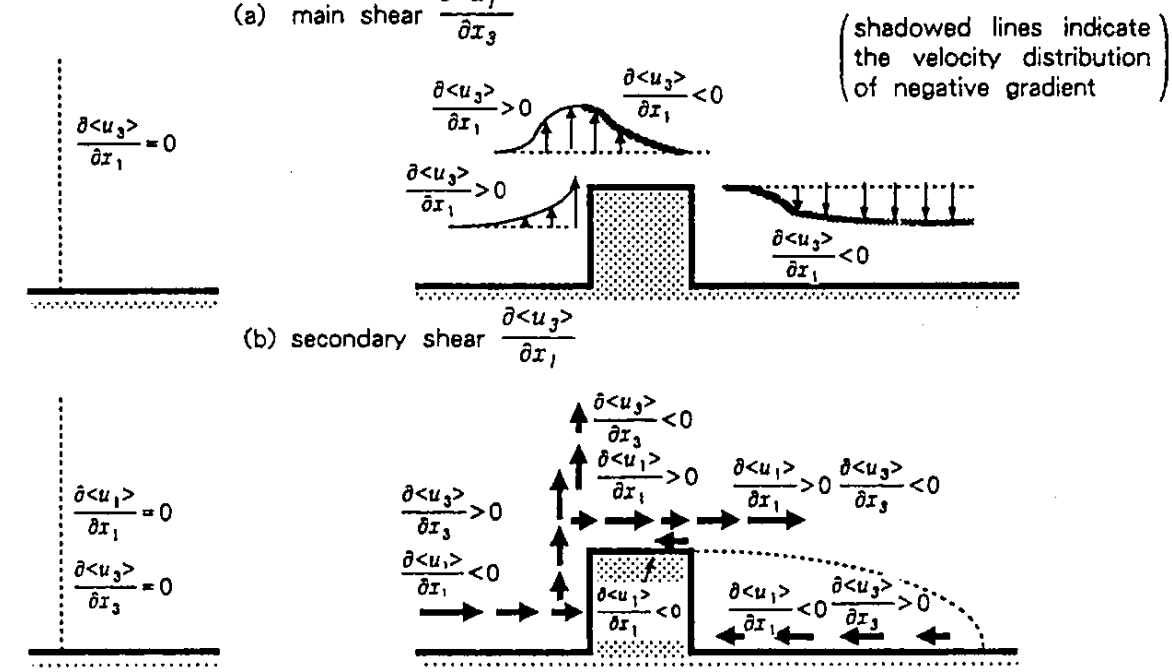

(b) secondary shear $\frac{\partial\left\langle u_{3}\right\rangle}{\partial x_{1}}$
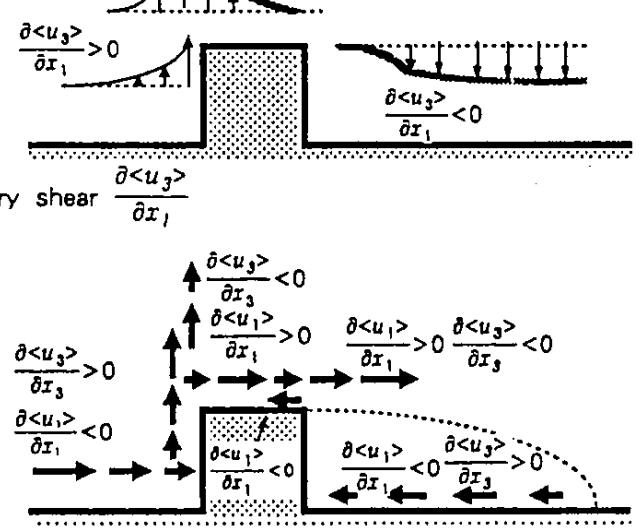

(c) diagonal components $\frac{\partial\left\langle u_{1}\right\rangle}{\partial x_{1}}, \frac{\partial\left\langle u_{3}\right\rangle}{\partial x_{3}}$

(1) Simple boundary layer (2) Flowfield around a $2 D$ square rib

Fig. 4. Distribution of velocity gradients around a simple bluff body. $x_{i}$ : three components of spatial coordinates $\left(i=1,2,3\right.$ : streamwise, lateral, vertical); $u_{i}$ : three components of wind velocity; $<>$ : ensemble average. (Murakami, 1993). 
Increased plume size, reduced turbulence scales and wake sources smooth out plume meander in the obstacle array.

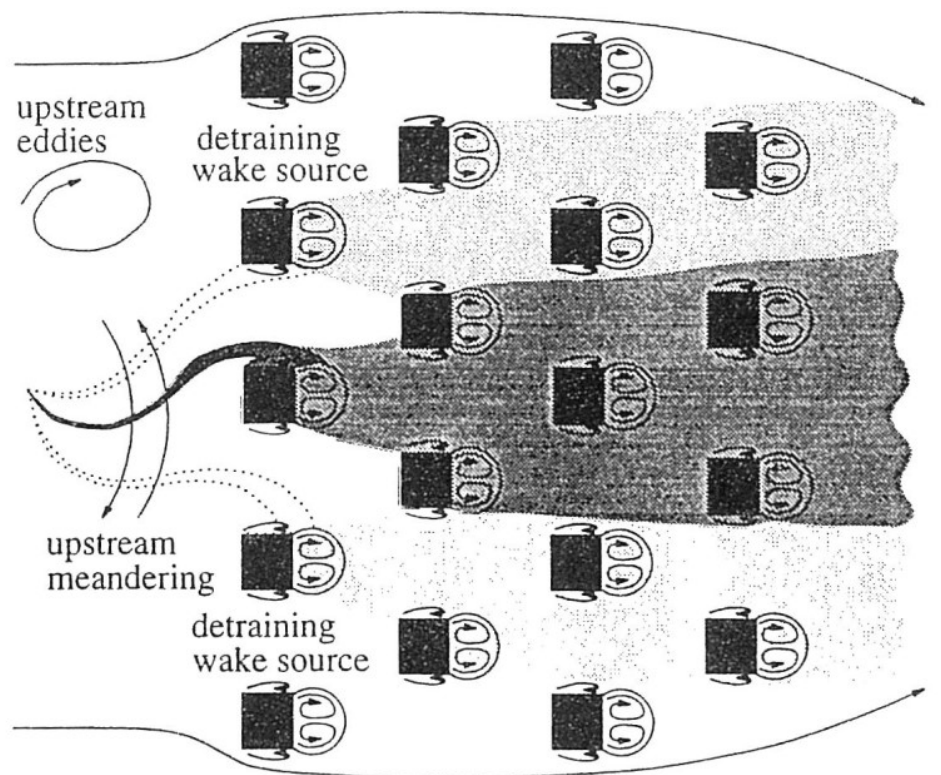

a) A plan view showing changes to the lateral extent of the array plume

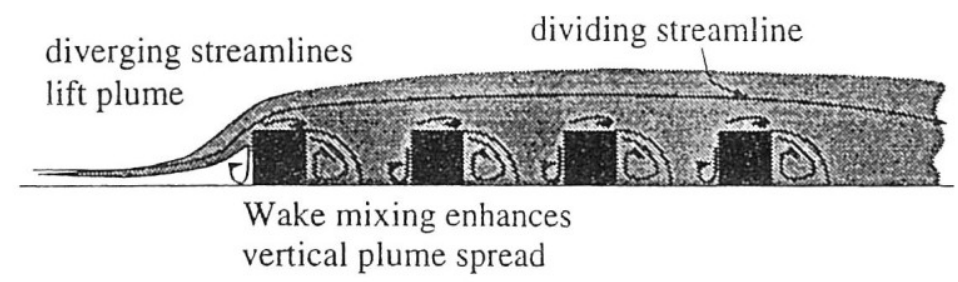

b) An elevation view showing changes to the vertical extent of the array plume

Fig. 5. Schematic diagram of plume dispersion through an array of obstacles (Davidson et al., 1995).

\subsection{Unsteadiness of large-scale flow structure}

Another characteristic of a flow field around a single building or a group of buildings is the high unsteadiness caused by the oncoming unsteady flow and its interaction with the wake flow connected with the process of vortex shedding. This can be a factor that influences pollutant dispersion in the urban area and causes complex behavior due to advection and turbulent diffusion.

A large-scale organized motion in the flow field around a bluff body can be explained easily for the flow past a $2 \mathrm{D}$ cylinder. Indeed, the time-varying flow in the wake of a $2 \mathrm{D}$ cylinder includes a periodic component distinguished from a residual 'random' ('turbulent') component (Lyn et al., 1995). This is conceptually illustrated in Fig. 6 (Murakami and Mochida, 1995), which shows that the periodic fluctuation organized by the large-scale flow structure should be specifically distinguished from the stochastic turbulent fluctuation. When an isolated bluff body has a simple shape, the contribution of the large scale unsteadiness, like vortex shedding, is relatively large. Alternatively, when the bluff body has a complicated shape, like an actual urban building complex, the stochastic turbulent fluctuation will be dominant. As described in the previous section, the flow field around buildings is characterized by the appearance of various types of vortices, i.e. a horseshoe vortex, an arc-shaped vortex, flow 
separation and vortex shedding. In fact, these large-scale vortices repeat converging/diverging and unsteadily interact. Such unsteady dynamics and organized structures over idealized three-dimensional arrays of buildings are clearly elucidated based on comprehensive data from DNS (Coceal et al., 2007; Branford et al., 2011). It should be emphasized that this type of large-scale unsteady fluctuations cannot be reproduced by steady RANS computations, in which time-derivative terms in the basic equations are equal to zero by considering the solution as being steady in time. However, this is not meant all RANS cannot reproduce it. Because RANS is derived by ensemble (Reynolds) average, it is basically applicable to non-stationary flows such as periodic or quasi-periodic flows involving deterministic structures. This approach is called as unsteady-RANS (URANS). However, most of the turbulence models used to close the equations are valid only as long as the time over which these changes in the mean occur is large compared to the time scales of the turbulent motion containing most of the energy. Therefore, special attention to turbulence models used is necessary to use URANS successfully. Currently URANS is hardly applied to the near-field dispersion problem around buildings, as will be discussed later in this paper.

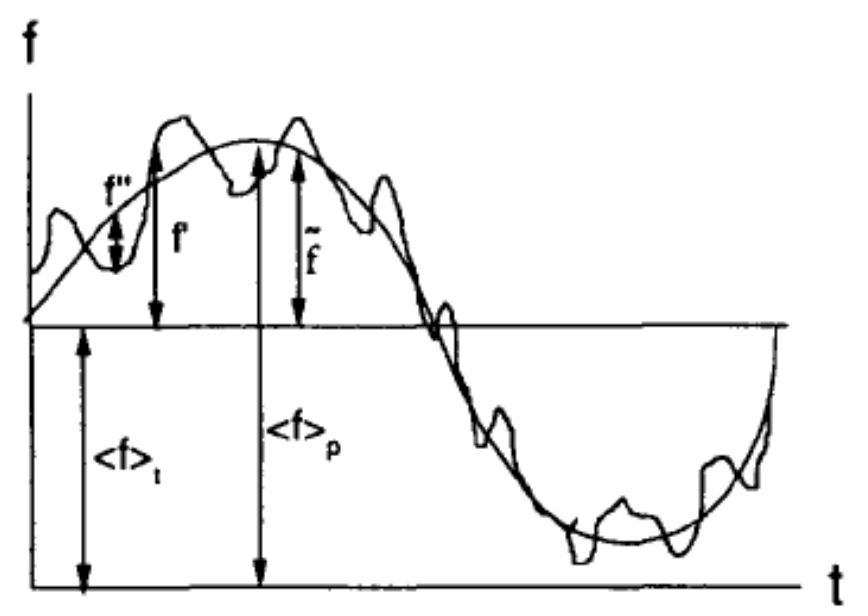

Fig. 6. Periodic and stochastic fluctuations in the turbulent vortex shedding flow. $\widetilde{f}$ : periodic fluctuation; $f^{\prime \prime}$ : stochastic turbulent fluctuation; $f^{\prime}$ : total fluctuation (periodic + stochastic); $\langle f\rangle_{p}$ : phase-averaged value; $\langle f\rangle_{i}$ : time-averaged value (Murakami and Mochida, 1995).

\subsection{Anisotropy of turbulent scalar fluxes}

For transport and dispersion around buildings, large eddies act to physically transport contaminants from one point in space to another. For example, a pollutant released in a street canyon is intermittently ejected out of the canyon due to the instantaneous flow field, and it results in periods of low and high concentration in the canyon. This 'non-local' mixing is not adequately accounted for the standard gradient diffusion hypothesis (SGDH) (Brown 2004). The need for advanced models for scalar fluxes and the limitations of the SGDH have been frequently reported and discussed since the comprehensive review by Launder (1978). Several algebraic formulations for the turbulent scalar flux have been proposed and some of them applied successfully to turbulent mass transfer in complex flows (Rogers et al., 1989; Abe and Suga, 2001; Younis et al., 2005). Recently, Rossi and Iaccarino (2009) showed that the SGDH failed to predict the streamwise component of the scalar flux through a numerical study of a line source downstream of a square obstacle. Rossi et al. (2010) concluded that algebraic closures, which can close the scalar flux term with a tensorial turbulent diffusivity, provide a significant improvement from the analysis using the DNS database for scalar dispersion downstream of a wall-mounted cube. Gousseau et al. (2011b) indicated that the counter-gradient mechanism occurs for not only cubic buildings, but also when the source is higher and less affected by the building-generated turbulence. It has been also demonstrated that large differences can be observed between the distributions of the estimated eddy viscosity by using LES data and the eddy diffusivity obtained by RANS for a building array model with a point source, since the eddy diffusivity is not always proportional to the eddy viscosity (Tominaga and Stathopoulos, 2012). This limitation of the SGDH is related to the problem of the turbulent Schmidt number in RANS computations as mentioned later. 
Prediction accuracy of various turbulence models

In terms of the accuracy of CFD simulations, the main sources of errors and uncertainties should be identified. The ERCOFTAC guidelines categorize errors and uncertainties in CFD simulations as model uncertainty, discretization or numerical error, iteration or convergence error, round-off errors, application uncertainties, user errors and code errors (Casey \& Wintergerste, 2000). The model uncertainty, which is due to the difference between the real flow and the exact solution of the model equations, is mainly discussed in this paper. As ensured in the ERCOFTAC guidelines, it should be noted that the relevance of turbulence modeling only becomes significant in CFD simulations when other sources of error, in particular the numerical and convergence errors, have been removed or properly controlled.

\subsection{Comparison of various RANS models}

In several studies, various RANS models have been compared for modeling dispersion fields around buildings (Meroney et al., 1999; Chan et al., 2002; Nazridoust and Ahmadi, 2006; Blocken et al., 2008; Tominaga and Stathopoulos, 2009). It is well known that the standard k- $\varepsilon$ model (SKE; Launder and Spalding, 1972) poorly represents separation flow due to the overestimation of turbulent kinetic energy (TKE) near the upwind corner of a building (Murakami, 1993). Therefore, when a source exit exists in the recirculation regions on the roof and walls, poor concentration prediction is observed. The SKE performance is improved by modifying k- $\varepsilon$ models, such as the RNG k- $\varepsilon$ model (RNG; Yakhot et al., 1992), on concentration prediction (Meroney et al., 1999; Tominaga and Stathopoulos, 2009; Blocken et al., 2011). However, when the exit is installed in other positions (e.g., elevated stacks or wake regions) the SKE sometimes shows a better result in comparison with the modified models (Wang and McNamara, 2006; Gousseau et al., 2011b). This is why the overestimation of TKE near the upwind corner in SKE causes large turbulent diffusion at the side and leeward walls of the building. The overestimation of turbulent diffusion observed in SKE counteracts the under-estimation of turbulent diffusion due to the lack of large-scale unsteady motion in RANS computation (Tominaga and Stathopoulos, 2010). The differences of the results between SKE and the modified k- $\varepsilon$ models are rather small for dispersion in street canyons and building complexes, where turbulence produced by surrounding buildings is dominant (Chang and Meroney, 2001; Nazridoust and Ahmadi, 2006). The Reynolds-stress models (RSM; Launder et al., 1975) has often provided the worst results in comparative studies of various turbulence models, although it can occasionally capture the near-wall flow phenomena (Murakami et al., 1996; Wang and McNamara, 2006; Koutsourakis et al., 2012). This is mainly because RSM requires optimization of many numerical parameters due to the comparatively high number of differential equations to be solved, and the simulations generally have a higher dependency on the chosen mesh and more difficulty converging when compared to the k- $\varepsilon$ models. However, RSM can account for various effects of complex turbulent flows in a rigorous manner. Therefore, the RSM needs further investigation for application to near-field pollutant dispersion around buildings, though $\mathrm{k}-\varepsilon$ models are presently ascendant by their robustness for various flow fields. In short, it is important to use turbulence models with a thorough understanding of their characteristics and limitations.

\subsection{Optimization of turbulent Schmidt number}

In the k- $\varepsilon$ type RANS models, the scalar flux is estimated using the SGDH with the eddy diffusivity typically being expressed by the eddy viscosity and the turbulent $\mathrm{Schmidt}$ number, $\mathrm{Sc}_{\mathrm{t}}$. Before discussing $\mathrm{Sc}_{\mathrm{t}}$, it should be reminded that SGDH is an inadequate model for a complex flow and various improved models have been proposed, as mentioned in section 3.3. Introducing $\mathrm{Sc}_{t}$ is, so to speak, a compromising method corresponding to the simplicity of the turbulence modeling in the k- $\varepsilon$ models. As pointed out by Tominaga and Stathopoulos (2007), the optimum values of $\mathrm{Sc}_{\mathrm{t}}$ provided in previous studies are sometime much different from the commonly used values of 0.7 to 0.9 and are widely spread from 0.2-1.3 according to the various flow properties and geometries. $\mathrm{Sc}_{\mathrm{t}}$ can be determined by measurements and much research on its values has been conducted including the turbulent Prandtl number over the years (Launder, 1978; Koeltzsch 2000; Flesch et al. 2002). According to these references, $\mathrm{Sc}_{\mathrm{t}}$ sometimes varies widely. Koeltzsch (2000) showed a strong height gradient in $\mathrm{Sc}_{\mathrm{t}}$, with a peak near one-third the boundary layer depth. The wide range of $\mathrm{Sc}_{\mathrm{t}}$ in measurement implies that $\mathrm{Sc}_{\mathrm{t}}$ has functional relationship to local flow properties. Therefore, optimization of $\mathrm{Sc}_{\mathrm{t}}$ should be performed based on local flow properties, and never be adjusted to correct for error in the mean flow calculation as it is a scalar diffusion parameter. Recently, Mokhtarzadeh-Dehghan et al. (2012) found that the mass eddy diffusivity needed to be reduced by increasing $\mathrm{Sc}_{\mathrm{t}}$ with dependence on Richardson number in order to model the experimentally observed spreading rates of heavier-than-air gases correctly. 
As demonstrated in many previous studies (Di Sabatino et al., 2007; Blocken et al., 2008; Gousseau et al., 2011a, b), smaller values of $\mathrm{Sc}_{t}$ often provide better results in comparison with experimental values. These results can be interpreted that underestimation of turbulent diffusion of momentum, which is often observed in steady-RANS computation (Tominaga et al. 2008a), is compensated by a smaller value of $\mathrm{Sc}_{\mathrm{t}}$. Chavez et al. (2011) verified that pollutant dispersion from a rooftop stack of an isolated building is greatly influenced by the value of $\mathrm{Sc}_{\mathrm{t}}$, however, in the presence of adjacent buildings changes in $\mathrm{Sc}_{\mathrm{t}}$ do not have a major impact on plume dilutions, especially downstream from the stack. This can be interpreted to imply that turbulent mixing enhanced by the surrounding buildings obscures the underestimation of turbulent diffusion in RANS computation. Moreover, this type of compensation can be found in discrepancy of the SGDH in modeling of a turbulent scalar flux. The SGDH cannot express anisotropy of turbulent scalar flux as discussed in the previous section. This type of inherent discrepancy can also be compensated by changing the value of $\mathrm{Sc}_{\mathrm{t}}$. For example, two values of $\mathrm{Sc}_{\mathrm{t}}$ could be used to ensure that the diffusivities for lateral and vertical directions are different. Simoes and Wang (1997) found that the best results were achieved with the anisotropic $\mathrm{Sc}_{\mathrm{t}}$ arrangement of 0.5 for horizontal mixing and 1.0 for vertical mixing for a compound channel flow (Shiono et al., 2003). Ideally, these discrepancies from turbulence modeling should be remedied by improving or upgrading turbulence modeling itself.

\subsection{Comparison of RANS and LES}

It is well known that in contrast to RANS, the LES resolves large-scale unsteady motions and requires only small-scale modeling. Therefore, dynamic properties such as the fluctuations of wind pressure on buildings, which are primarily due to large-scale motions, can be directly reproduced in LES. Furthermore, several studies have argued that the results of LES show good agreement with experimental data in terms of distributions of mean velocity and turbulent energy around a simple building, even when the simple sub-grid scale model was used (Murakami, 1993; Rodi, 1997; Tominaga et al., 2008a). This is because the momentum diffusion due to large scale fluctuations - say vortex shedding around a building, which is not reproduced in steady-RANS computation - is closely related to the formation of the flow structure around a bluff body.

According to previous studies comparing RANS and LES for dispersion modeling around buildings (Xie and Castro, 2009; Dejoan et al., 2010; Santiago et al., 2010; Tominaga and Stathopoulos, 2010, 2011, 2012; Salim et al. 2011, Gousseau et al., 2011a, b), LES gives better results than RANS for calculating the distribution of concentration, although the difference between LES and RANS results for mean velocity is not as large. This is because the horizontal and vertical diffusions of concentration are reproduced well by LES, due mainly to the reproduction of unsteady concentration fluctuations around the building in comparison to RANS computations. Fig. 7 illustrates the contours of normalized concentrations on building surfaces and surrounding streets from the rooftop stack on the central building in Montreal by RANS SKE and LES (Gousseau et al., 2011a). With both models, the maximum concentrations occur on the leeward facade of the tall building but the concentration values on the building surfaces and the surrounding streets predicted with LES are overall lower than those predicted with SKE. The concentration obtained by LES is much diffusive in horizontal and vertical directions. By comparing with the experimental data, LES results are more accurate than those from RANS SKE. Furthermore, Moonen et al. (2012) found that flow unsteadiness has a significant effect on the performance of air exchange in an idealized urban environment by comparison between steady RANS and LES.

As mentioned above, the turbulent mass diffusion attributed to the large-scale structures that emanate from the leading edge of the buildings is too complex to be modeled adequately by using SGDH. Therefore, only an unsteady state computation, such as LES, is capable of reproducing the complicated turbulent mass transfer that occurs around buildings. However, the CPU time required to obtain the statistical values in LES is 10-25 times longer than that in corresponding steady-RANS computations; although it is difficult to compare the computational time of LES and RANS methodologies directly due to the different convergence criteria used by the two methods. In this sense, unsteady-RANS (URANS) computation is promising, because URANS can reproduce large-scale unsteady structures with lower spatial and temporal resolutions than LES. However, there are very few URANS examples investigating flow around a building (Iaccarino et al., 2003; Shao et al., 2012). Iaccarino et al. (2003) demonstrated that URANS predicted periodic shedding and led to much better concurrence with available experimental data than has been achieved with steady computation for flow over a wall-mounted cube. It should be noted here that URANS only resolves the fluctuations due to the large-scale flow structure, while it models the turbulence fully. On the other hand, LES actually resolves most scales of the turbulence. Therefore, URANS is more successful for flows whose unsteadiness is deterministic, such as vortex shedding in the wake of an obstacle with a low-turbulence approach flow (Kato and Launder, 1993; Shimada and Ishihara, 2002). In order to apply URANS to relatively high turbulence approach flows such as atmospheric boundary layer flows, it is necessary to appropriately model the interaction 
between the organized large-scale flow unsteadiness and the random turbulence motions in the turbulence model (Younis and Zhou, 2006).

LES computation can yield important information on instantaneous fluctuations of concentration that cannot be directly obtained by RANS computations. However, in compensation, a model solving the transport equation of the concentration variance has been known as the prediction method of concentration fluctuation in RANS model (Launder, 1978). With the availability of measurements of concentration fluctuation in a plume dispersing in an urban area, efforts to model the concentration variance for urban plumes have been undertaken by several researchers (Andronopoulos et al., 2002; Hsieh et al., 2007; Mavroidis et al., 2007; Wang et al., 2009; Milliez and Carissimo, 2008; Yee et al., 2009). Generally, it has been demonstrated that these models can be used to reproduce concentration variance in an idealized urban area under laboratory conditions. However, the models for closure of the transport equations for concentration variance need to be further validated using other available urban flow and pollutant dispersion data from both laboratory and full-scale experiments.
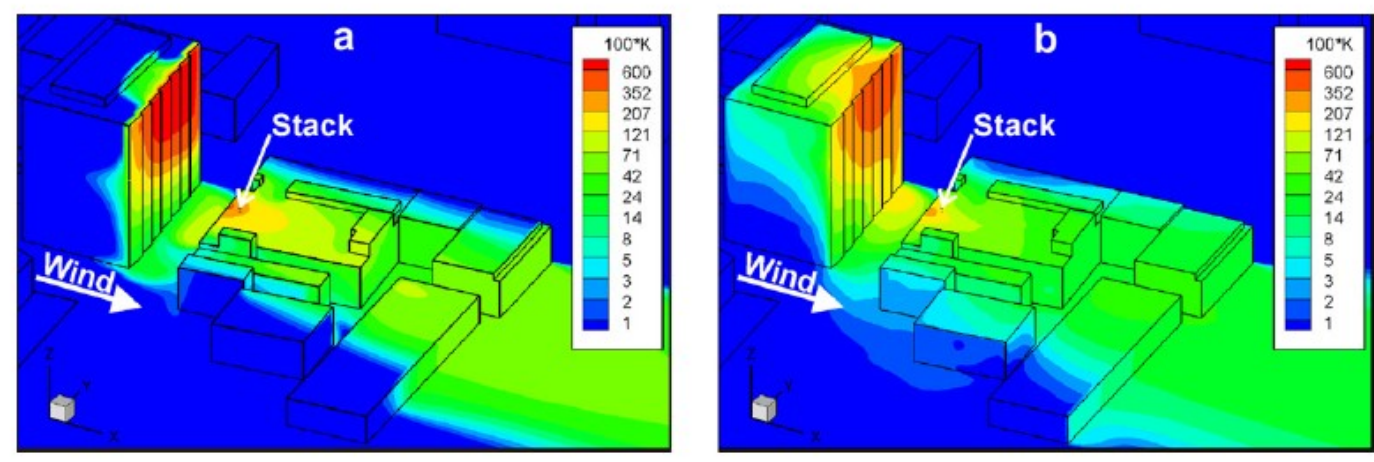

Fig. 7. Contours of normalized concentrations $(\mathrm{K})$ on building surfaces and surrounding streets for different turbulence models; (a) SKE and (b) LES (Gousseau et al., 2011a).

\section{5}

\section{Issues with modeling of boundary conditions}

The accuracy of CFD is also affected by uncertainties in the precise geometry, uncertain data or models that need to be specified as boundary conditions such as turbulence properties at an inlet (Casey \& Wintergerste, 2000). This section describes two important issues with modeling of boundary conditions, i.e., inflow boundary condition and expression of unresolved obstacles, affecting near-field pollutant dispersion in the urban environment.

\subsection{Inflow boundary condition}

As mentioned previously, pollutant dispersion around buildings is characterized by the complex interaction between atmospheric boundary flow and flow around buildings. Therefore, accurate simulation of the atmospheric boundary flow in the computational domain is imperative to obtain accurate and reliable predictions of the intended dispersion processes. In order to realize this, sufficient fetch where the boundary layer development is slow enough and consistency between the ground surface boundary condition and the fully-developed inlet profiles are required. For fetch distance, Robins (1979) concluded that a fetch of about 6-7 times as long as the boundary layer depth is needed for development. For the consistency between the well-used sand-grain based rough-wall function in RANS computations and the fully-developed inlet profile, several remedial measures have been proposed (Blocken et al., 2007; Hargreaves and Wright, 2007; Yang et al., 2009; Parente et al., 2011).

Furthermore, the mean and turbulent properties corresponding to actual weather conditions need to be given adequately at an inflow boundary. In RANS computations, this is comparatively simple since turbulent statistics can directly be given at the boundary (Kondo et al., 2006; Baik et al., 2009). On the other hand, LES is accompanied by difficulty, because the process needs to be provided with physically appropriate temporal and spatial fluctuations. As widely recognized, the inflow turbulence has strong influence on the turbulent flow structure around buildings. Hence, the treatment of inflow turbulence is very important to obtain reliable results in LES. Much effort has been made to provide the suitable inlet conditions for LES over many years and results of the recent development in engineering fields can be found in an overall review by Tabor and Baba-Ahmadi (2010). Xie and Castro (2008) 
developed the synthetic method of turbulence generation in order to implement dynamic spatial boundary conditions derived from the unsteady output of much larger-scale computations coupled with the new small-scale turbulence inflow method. Xie (2011) reported that the dispersion in an urban region was found sensitive to the variation of wind direction through LES of flow and dispersion in an oblique wind over the DAPPLE site.

Moreover, influence of the stability of inflow boundary layers on near-field dispersion is a much more complex topic in contrast to the neutral case. This is because the height of an internal boundary layer, which is generated by each local surface condition, grows with increasing fetch. The rate of the growth depends on the roughness and the stability. The influence of the atmospheric stability on dispersion in urban configurations has been investigated by wind tunnel and field experiments (Higson et al., 1995; Uehara et al., 2000; Santos et al., 2005; Yassin 2013). Meanwhile, several research studies have been conducted on the reproduction of the influence of atmospheric stability on near-field dispersion using CFD (Zhang et al., 1996; Baklanov, 2000; Santos et al. 2009; Xie, 2010; Cheng and Liu, 2011b; Mavroidis et al., 2012). As reported by Santos et al. (2009), stability conditions significantly affect plume spread and concentration levels around buildings by the intermediary of TKE level generated by temperature gradients. Recently, Xie et al. (2013) investigated thermal stratification effects of approach flows on dispersion over a group of staggered cubes and the DAPPLE site using LES, wind-tunnel and field experiments. They performed a number of numerical experiments including cases with various bulk Richardson number $\mathrm{R}_{\mathrm{b}}$ and concluded that stratification effects on dispersion in weakly unstable conditions are not negligible.

Additionally, some researchers also indicated that the influence of solar radiation has significant influence on pollutant diffusion inside street canyons (Sini et al. 1996, Smith et al., 2001; Xie et al., 2005). These studies reveal that the differential heating of street surfaces due to solar radiation can largely influence the flow's capability to transport and exchange pollutants.

\subsection{Unresolved obstacles}

Many obstacles affect near-field dispersion in urban environments. Generally, since it is difficult to explicitly resolve them all by grids used in CFD, some kind of modeling should be introduced. Canopy models have been developed for representing surface roughness effect of obstacles on wind profiles for a long time. Recently, the canopy models have widely been used in order to consider the aerodynamic effects of various unresolved obstacles. In the canopy models, extra terms designed to represent the drag and increased turbulence due to obstacles, are added to governing equations. For example, trees are common obstacles in urban environment. The presence of trees induces a decrease in wind velocity and changes in TKE. In the literature, several canopy models have been developed in order to model the forested areas effects, with different formulations for additional source terms in TKE and dissipation rate equations (Svensson and Häggkvist 1990; Green 1992; Hiraoka, 1993; Liu et al. 1996; Sanz 2003; Katul et al. 2004; Mochida et al. 2008). Mochida et al. (2008) optimized the model coefficients used in the tree canopy models by comparing numerical results with field measurements of wind velocity and TKE around trees. The influence of trees on pollutant diffusion inside street canyons has been investigated both experimentally (Gromke et al., 2007; 2009) and by numerical models (Gromke et al., 2008; Buccolieri et al., 2009; 2011).

Furthermore, the modeling of traffic, which is the main pollution source in a street, is also important. Effects of moving vehicles on dispersion around street canyons have been investigated experimentally (Baker and Hargreaves, 2001; Ahmad et al., 2002). As pointed out by Baker and Hargreaves (2001), there is a large variability between individual concentration time series with moving vehicles in wind tunnel, because of a normal feature of the turbulent wind and vehicle wake system where the turbulence length scale is greater than the vehicle/wake scale. Therefore, ensemble averaging is required to evaluate the concentration. The ensemble-averaged properties in experiments can only be compared with RANS CFD results. A 3-D Eulerian-Lagrangian approach to moving vehicles that takes into account the traffic-induced flow and turbulence was proposed by Jicha et al. (2000) for pollutant dispersion in a street canyon. Mochida and Lun (2008) extended the methodology of the canopy model to incorporate the aerodynamic effect of the moving vehicles based on the $\mathrm{k}-\varepsilon$ model, in which extra terms were added in the transport equations. On the other hand, an explicit treatment of moving vehicles can be undertaken by computations using the moving finite element/control volume method (e.g. Fluidity, 2012). This method allows arbitrary movement of the mesh with time dependent problems like a passing vehicle. Venetsanos et al. (2002) studied the impact of various factors that may affect the exhaust plume dispersion, different driving environments, car speeds and meteorological conditions, by CFD computations performed in a moving coordinate system with the car and site geometry being fully resolved.

\section{Model evaluation}


Validation process is an essential part of model evaluation to provide qualitative and quantitative views of the model (Blocken and Gualtieri, 2012). For many users, it is most important to determine the accuracy and confidence of the model results. Unfortunately, it is very difficult to determine how to validate based on accuracy because pollutant concentrations around buildings can vary by orders of magnitude in time and space. For comparison with wind tunnel data, the German VDI Guideline on wind field models (VDI, 2005) requires a certain hit rate for a number of specific test cases. For example, for wind components at specific points behind a building, the guideline requires a hit rate of $\mathrm{q}>66 \%$, with an allowed deviation $\mathrm{D}=0.25$. Other typical values are given by Chang \& Hanna (2004). Recently, based on accumulated knowledge from the meteorological field, the COST Action 732 (2005-2009) proposed and tested a new protocol for evaluation and quality assurance of numerical models for micro-scale urban meteorology (Britter and Schatzmann, 2007). This document assists in the establishment and execution of a model evaluation exercise for the use of microscale meteorological models in the prediction of the flow and/or the dispersion of pollutants within and near an urban canopy layer. The proposed evaluation protocol has several distinct elements: a scientific evaluation process, a verification process, the provision of appropriate and quality assured validation data sets, a model validation process, and an operational evaluation process that reflects the needs and responsibilities of the model user. Issues with the validation of urban flow and dispersion CFD models from a meteorological viewpoint have been overviewed by Schatzmann et al. (1997) and Schatzmann and Leitl (2002; 2011).

It is also important to determine which variables should be used for evaluation. The maximum concentration is a key metric for most air quality applications. However, low values of concentration need to be accurately calculated because they may significantly affect population exposure assessments. For example, the lethality of low concentrations may not be as great, but because the dilute plume covers a greater area, it can still affect many people. Furthermore, accurate prediction of plume location and coverage is very important when developing and evaluating urban dispersion models, but it poses a difficult challenge (Brown, 2004). From this viewpoint, statistical analysis based on various metrics recommended in previous studies (Chang and Hanna, 2004; COST Action 732, 2009) has limitations in use for evaluating plume location or coverage; this type of analysis is helpful in estimating the errors in models, but it can lead to misleading conclusions due to the limited experimental data set (Santiago et al., 2010).

Time-averaged concentration, which is relatively easy to obtain in experiments, is often used for evaluations. However, concentration fluctuations around buildings are very important, particularly when plume contains toxic, flammable, or odorous components. The prediction results are often required in terms of percentile exceedances, rather than absolute maximum results. This makes the results more robust, and probably more realistic for assessment manners. In such cases, LES, which can directly evaluate a specific percentile of concentration, has a great advantage when compared to the RANS approach. When using LES, it should be noted that evaluation time for obtaining statistical average values is quite important (Schatzmann and Leitl, 2011; Harms et al., 2011).

\subsection{Measurements data for validation}

In order to perform high quality validation and uncertainty evaluation, reliable experimental data are indispensable. In particular, it is important that the required boundary conditions, such as inflow conditions, are carefully provided for computations. As pointed out by Schatzmann and Leitl (2011), atmospheric variability may be critical when CFD results are compared with field measurement data. Since field experiments are generally treated as a set of individual realisations, sufficiently large ensembles of observations over the range of external parameters, such as wind direction, should be obtained to provide useful statistical information. In this sense, very few dispersion studies have ever really produced sufficiently large datasets to meet this condition. The constraint is felt particularly strongly in short-range urban dispersion because of the high degree of inherent natural variability, coupled with confounding factors such as traffic conditions (Belcher et al., 2012). Therefore, it is recommended that validation data based on a combination of field and boundary layer wind tunnel experiments are provided.

Several experimental datasets based on wind tunnel tests are available on the internet. For example, CEDVAL (2002), which was provided at the Meteorological Institute, Hamburg University, is a compilation of mainly wind tunnel datasets that can be used for validation of numerical dispersion models. All datasets within CEDVAL follow a high quality standard in terms of complete documentation of boundary conditions and quality assurance during measurements. The database will be extended step by step by more complex configurations. On the other hand, the DAPPLE project is mostly a field-based campaign targeted at central London, aiming to understand the influence of urban traffic and urban junction topographies on the dispersion of pollutants (Arnold et al., 2004; Wood et al., 2009). Detailed wind tunnel experiments were also performed in order to study flow and pollutant dispersion in the real 
urban environment (Carpentieri et al., 2009). The experimental set up allowed the measurement of flow and dispersion around a small-scale model of the DAPPLE site, as well as the estimation of mean and turbulent fluxes in the urban canopy (Carpentieri and Robins, 2010; Carpentieri et al., 2012). In this study, comprehensive and detailed flow and dispersion datasets were collected that were suitable for model evaluation work at all levels from the empirical model to CFD (DAPPLE Datasets, 2008). It is expected that the reliability of CFD models will be clarified and established by performing cross comparisons using these datasets.

\section{Conclusions}

This article documented the current modeling and computational methods in CFD simulations of near-field pollutant dispersion around buildings in order to give a perspective on CFD modeling and give some insights into future directions for practical applications. First, previous CFD studies of near-field pollutant dispersion around buildings, conducted with different research purposes, configurations, boundary conditions, and modeling approaches, were reviewed. These delivered an overview of CFD applications to near-field pollutant dispersion in the urban environment in recent years. Next, key features of near-field pollutant dispersion around buildings from previous studies, i.e., three-dimensionality of mean flow, unsteadiness of large-scale flow structure, and anisotropy of turbulent scalar fluxes, were identified and discussed. This review highlights that it is important to choose appropriate numerical models and boundary conditions by understanding their inherent strengths and limitations. Finally, the importance of model evaluation was emphasized. Since pollutant concentrations around buildings can vary by orders of magnitudes in time and space, the model evaluation should be performed carefully, while paying attention to their uncertainty. It was reconfirmed that the quantification of uncertainty was a cornerstone activity of any verification and validation program.

Applications of CFD to near-field pollutant dispersion around buildings have been rapidly increased in recent years, as presented in this review. Furthermore, its range is widely expanded to include various factors such as plume with strong negative and positive buoyancies, chemical reactions and fine particle dispersions. Clearly, CFD has considerable potential, as demonstrated by recent scientific accomplishments in this area. However, in order to use it appropriately, sufficient attention to the model theory and implementation is required. A previous review paper on past achievements and future challenges in Computational Wind Engineering (CWE) concluded with the following remarks (Stathopoulos, 2002):

"In spite of some interesting and visually impressive results produced with Computational Wind Engineering, the numerical wind tunnel is still virtual rather than real. Its potential however, is extremely high and its progress should be monitored carefully. Many more parallel studies - numerical and experimental - will be necessary in order to increase the present level of confidence in the computational results. Practitioners should be warned about the uncertainties of the numerical wind tunnel results and urged to exercise caution in their utilization. Finally, more effective efforts should be made in the numerical simulation of fluctuating flow field and the numerical evaluation of peak values of variables necessary for design."

In the past decade, CFD has become a more accessible tool due to the continued progress of modeling studies and the rapid increase of computational resources. In particular, for the prediction of the pedestrian wind environment, CFD has already been a viable method using several practical guidelines. However, for near-field pollutant dispersion around buildings, it is still needed to discern the applicability of CFD further with paying careful attention to the above quote. As demonstrated in this paper, inevitably, high quality CFD is often time consuming and costly. The validity of the level of expertise required and the time (cost) involved should be carefully evaluated on the basis of its purposes by comparing them with those of other assessment methods.

\section{Acknowledgements}

The framework of this paper was developed during the period that the first author worked as a visiting scientist in the Department of Building, Civil and Environmental Engineering of Concordia University, Montreal, Canada in August 2011. The authors would like to express their gratitude to FY 2011 Researcher Exchange Program between JSPS (Japan Society for the Promotion of Science) and NSERC (Natural Sciences and Engineering Research Council of Canada) for supporting this visit. This work was also financially supported by the Grants-in-Aid for Scientific Research in Japan (No. 21560622). Sincere thanks also go to the reviewers for their valuable comments and suggestions.

\section{References}


Abe, K., Suga, K., 2001. Toward the development of a Reynolds-averaged algebraic turbulent scalar flux model. International Journal of Heat and Fluid Flow 22, 19-29.

Ahmad, K., Khare, M., Chaudhry, K.K., 2002. Model vehicle movement system in wind tunnels for exhaust dispersion studies under various urban street configurations. Journal of Wind Engineering and Industrial Aerodynamics 90, 1051-1064.

Ahmad, K., Khare, M., Chaudhry, K. K., 2005. Wind tunnel simulation studies on dispersion at urban street canyons and intersections-a review. Journal of Wind Engineering and Industrial Aerodynamics 93, 697-717.

Allwine, K.J., 2004. Overview of JOINT URBAN 2003 an atmospheric dispersion study in Oklahoma City. Proceedings of the AMS Symposium on Planning, Nowcasting, and Forecasting in the Urban Zone, January 11-15, Seattle, Washington, USA .

Andronopoulos, S., Grigoriadis, D., Robins, A., Venetsanos, A., Rafailidis, S., Bartzis, J.G., 2001. Three-dimensional modelling of concentration fluctuations in complicated geometry. Environmental Fluid Mechanics 1, 415-440.

Antonioni, G., Burkhart, S., Burman, J., Dejoan, A., Fusco, A., Gaasbeek, R., Gjesdal, T., Jäppinen, A., Riikonen, K., Morra, P., Parmhed, O., Santiago, J.L., 2012. Comparison of CFD and operational dispersion models in an urban-like environment. Atmospheric Environment 47, 365-372.

Arnold, S., ApSimon, H., Barlow, J., Belcher, S., Bell, M., Boddy, D., Britter, R., Cheng, H., Clark, R., Colvile, R., Dimitroulopoulou, S., Dobre, A., Greally, B., Kaur, S., Knights, A., Lawton, T., Makepeace, A., Martin, D., Neophytou, M., Neville, S., Nieuwenhuijsen, M., Nickless, G., Price, C., Robins, A., Shallcross, D., Simmonds, P., Smalley, R., Tate, J., Tomlin, A., Wang, H., Walsh, P., 2004. Dispersion of Air Pollution \& Penetration into the Local Environment - DAPPLE. Science of the Total Environment 332, 139-153.

ASHRAE, 2007. Building air intake and exhaust design, ASHRAE applications handbook, American Society of Heating, Refrigerating and Air-Conditioning Engineering Inc., Atlanta, USA, Chapter 44.

ASHRAE, 2011. Building air intake and exhaust design, ASHRAE handbook: HVAC Applications (SI), American Society of Heating, Refrigerating and Air-Conditioning Engineers, Inc., Atlanta, USA, Chapter 45.

Baik, J.-J., Kim, J.-J., 1999. A numerical study of flow and pollutant dispersion characteristics in urban street canyons. Journal of the Applied Meteorology 38, 1576-1589.

Baik J.-J.; Kim J.-J., 2002. On the escape of pollutants from urban street canyons. Atmospheric Environment 36, 527-536.

Baik, J.-J., Kim, J.-J., Fernando, H.J.S., 2003. A CFD model for simulating urban flow and dispersion. Journal of Applied Meteorology 42, 1636-1648.

Baik, J.-J., Park, S.-B., Kim, J.-J., 2009. Urban flow and dispersion simulation using a CFD model coupled to a mesoscale model. Journal of Applied Meteorology and Climatology 48, 1667-1681.

Baker, C.J., Hargreaves, D.M., 2001. Wind tunnel evaluation of a vehicle pollution dispersion model. Journal of Wind Engineering and Industrial Aerodynamics 89, 187-200.

Baklanov, A., 2000. Application of CFD methods for modelling in air pollution problems: possibilities and gaps. Journal of Environmental Monitoring and Assessment 65, 181-190.

Belcher, S.E., 2005. Mixing and transport in urban areas. Philosophical Transactions of the Royal Society A 363, 2947-2968.

Belcher, S. E., Coceal, O., Hunt, J. C.R., Carruthers, D. J., Robins, A. G., 2012. A review of urban dispersion modeling. UK Atmospheric Dispersion Modelling Liaison Committee.

Blocken, B., Stathopoulos, T., Carmeliet, J., 2007. CFD simulation of the atmospheric boundary layer: wall function problems. Atmospheric Environment 41, 238-252.

Blocken, B., Stathopoulos, T., Saathoff, P., Wang, X., 2008. Numerical evaluation of pollutant dispersion in the built environment: comparisons between models and experiments. Journal of Wind Engineering and Industrial Aerodynamics 96, 1817-1831.

Blocken, B., Stathopoulos, T., Carmeliet, J., Hensen, J.L.M., 2011. Application of CFD in building performance simulation for the outdoor environment: an overview. Journal of Building Performance Simulation 4, 157-184.

Blocken, B., Gualtieri, C., 2012. Ten iterative steps for model development and evaluation applied to Computational Fluid Dynamics for Environmental Fluid Mechanics. Environmental Modelling \& Software 33, 1-22.

Blocken, B., Janssen, W.D., Hooff, T.V., 2012. CFD simulation for pedestrian wind comfort and wind safety in urban areas: General decision framework and case study for the Eindhoven University campus. Environmental Modelling \& Software 30, 15-34.

Boppana, V.B.L., Xie, Z.-T., Castro, I.P., 2010. Large-eddy simulation of dispersion from surface sources in arrays of obstacles. Boundary-Layer Meteorology 135, 433-454. 
Branford, S., Coceal, O., Thomas, T.G., Belcher, S.E., 2011. Dispersion of a point-source release of a passive scalar through an urban-like array for different wind directions. Boundary-Layer Meteorology 139, 367-394.

Britter, R., Schatzmann, M., Eds., 2007. Model evaluation guidance and protocol document, COST, Brussels.

Brown, M.J., 2004. Urban Dispersion: Challenges for Fast Response Modelling. 5th AMS Symposium on the Urban Environment, Vancouver, B.C., August 2004.

Buccolieri, R., Gromke, C., Sabatino, S.D., Ruck, B., 2009. Aerodynamic effects of trees on pollutant concentration in street canyons. Science of the Total Environment 407, 5247-5256.

Buccolieri, R., Sandberg, M., Di Sabatino, S., 2010. City breathability and its link to pollutant concentration distribution within urban-like geometries. Atmospheric Environment 44, 1894-1903.

Buccolieri, R.., Salim, S.M., Leo, L.S., Di Sabatino, S., Chan, A., Ielpo, P., de Gennaro, G. and Gromke, C., 2011. Analysis of local scale tree-atmosphere interaction on pollutant concentration in idealized street canyons and application to a real urban junction. Atmospheric Environment 45, 1702-1713.

Cai, X.M., Barlow, J.F., Belcher, S.E., 2008. Dispersion and transfer of passive scalars in and above street canyons Large-eddy simulations. Atmospheric Environment 42, 5885-5895.

Carpentieri, M., Robins, A. G., Baldi, S., 2009. Three-dimensional mapping of air flow at an urban canyon intersection. Boundary-Layer Meteorology 133, 277-296

Carpentieri, M., Robins, A.G., 2010. Tracer flux balance at an urban canyon intersection. Boundary-Layer Meteorology 135, 229-242.

Carpentieri, M., Hayden, P., Robins, A. G., 2012. Wind tunnel measurements of pollutant turbulent fluxes in urban intersections. Atmospheric Environment 46, 669-674.

Carruthers, D.J., Holroyd, R.J., Hunt, J.C.R., Weng, W.-S., Robins, A.G., Apsley, D.D., Thompson, D.J., Smith, F.B., 1994. UK-ADMS: A new approach to modelling dispersion in the earth's atmospheric boundary layer. Journal of Wind Engineering and Industrial Aerodynamics 52, 139-153.

Carruthers, D.J., Mckeown, A.M., Hall, D.J., Porter, S., 1999. Validation of ADMS against wind tunnel data of dispersion from chemical warehouse fires. Atmospheric Environment 33, 1937-1953.

Casey, M., Wintergerste, T., eds., 2000. ERCOFTAC Special Interest Group on "Quality and Trust in Industrial CFD": Best Practice Guidelines. ERCOFTAC.

CEDVAL dataset, 2002. Hamburg University, http://www.mi.uni-hamburg.de/cedval

CERC, 2006. ADMS-Urban, User Guide, Available from Cambridge Environmental Research Consultant, Cambridge, UK.

Chan, T.L., Dong, G., Leung, C.W., Cheung, C.S., Hung, W.T., 2002. Validation of a two-dimensional pollutant dispersion model in an isolated street canyon. Atmospheric Environment 36, 861-872.

Chang, C.H., Meroney, R.N., 2001. Numerical and physical modeling of bluff body flow and dispersion in urban street canyons. Journal of Wind Engineering and Industrial Aerodynamics 89, 1325-1334.

Chang, C.H., Meroney, R.N., 2003. Concentration and flow distributions in urban street canyons: wind tunnel and computational data. Journal of Wind Engineering and Industrial Aerodynamics 91, 1141-1154.

Chang, J.C., Hanna, S.R., 2004. Air quality model performance. Meteorology and Atmospheric Physics 87, 167-196.

Chavez, M., Hajra, B., Stathopoulos, T., Bahloul, A., 2011. Near-field pollutant dispersion in the built environment by CFD and wind tunnel simulations. Journal of Wind Engineering and Industrial Aerodynamics 99, 330-339.

Cheng, W.C., Liu, C.H., 2011a. Large-eddy simulation of flow and pollutant transports in and above two-dimensional idealized street canyons. Boundary-Layer Meteorology 139, 411-437.

Cheng, W.C., Liu, C.H., 2011b. Large-eddy simulation of turbulent transports in urban street canyons in different thermal stabilities. Journal of Wind Engineering and Industrial Aerodynamics 99, 434-442.

Coceal, O., Dobre, A., Thomas, T.G., 2007. Unsteady dynamics and organized structures from DNS over an idealized building canopy. International Journal of Climatology 27, 1943-1953.

CODASC Database, 2008. Concentration Data of Street Canyon internet database. http://www.codasc.de.

COST Action 732, 2009. Quality Assurance and Improvement of Micro-Scale Meteorological Models. http://www.mi.uni-hamburg.de/Home.484.0.html

Cowan, I.R., Castro, I.P., Robins, A.G., 1997. Numerical considerations for simulations of flow and dispersion around buildings. Journal of Wind Engineering and Industrial Aerodynamics 67-68, 535-545.

DAPPLE Data-sets, 2008. http://www.dapple.org.uk/downloads.html

Davidson, M.J., Mylne, K.R., Jones, C.D., Phillips, J.C., Perkins, R.J., Fung, J.C.H., Hunt, J.C.R., 1995. Plume dispersion through large groups of obstacles-A field investigation. Atmospheric Environment 29, 3245-3256.

Davidson, M.J., Snyder, W.H., Lawson Jr, R.E., Hunt, J.C.R., 1996. Wind tunnel simulations of plume dispersion through groups of obstacles. Atmospheric Environment 30, 3715-3731. 
Dejoan, A., Santiago, J.L., Martilli, A., Martin, F., Pinelli, A., 2010. Comparison between large-eddy simulation and Reynolds-averaged Navier-Stokes computations for the MUST field experiment. Part II: Effects of incident wind angle deviation on the mean flow and plume dispersion. Boundary-Layer Meteorology 135, 133-150.

Delaunay, D., Lakehal, D., Barré, C., Sacré, C., 1997. Numerical and wind tunnel simulation of gas dispersion around a rectangular building. Journal of Wind Engineering and Industrial Aerodynamics 67-68, 721-732.

Dezs-Weidinger, G., Stitou, A., Beeck, J.V., Riethmuller, M.L., 2003. Measurement of the turbulent mass flux with PTV in a street canyon. Journal of Wind Engineering and Industrial Aerodynamics 91, 1117-1131.

Di Sabatino, S., Buccolieri, R., Pulvirenti, B., Britter, R., 2007. Simulations of pollutant dispersion within idealised urban-type geometries using CFD and integral models. Atmospheric Environment 41, 8316-8329.

Di Sabatino, S., Buccolieri, R., Olesen, H.R., Ketzel, M., Berkowicz, R., Franke, J., Schatzmann, M.K., Schlunzen, H., Leitl, B., Britter, R., Borrego, C., Margari, A., 2011. COST 732 in practice - the MUST model evaluation exercise. International Journal of Environment and Pollution, 44, 403-418.

Flesch, T.K., 2002. Turbulent Schmidt number from a tracer experiment. Agricultural and Forest Meteorology 111, 299-307.

Fluidity Manual Version 4.1, 2012. Applied Modelling and Computation Group (AMCG), Imperial College London, http://amcg.ese.ic.ac.uk.

Franke, J., Hirsch, C., Jensen, A.G., Krüs, H.W., Schatzmann, M., Westbury, P.S., Miles, S.D., Wisse, J.A., Wright, N.G., 2004. Recommendations on the use of CFD in wind engineering In: Proceedings of the International Conference on Urban Wind Engineering and Building Aerodynamics. In: van Beeck JPAJ (Ed.), COST Action C14, Impact of Wind and Storm on City Life Built Environment. von Karman Institute, Sint-Genesius-Rode, Belgium, 5-7 May 2004.

Franke, J., Hellsten, A., Schlünzen, H., Carissimo, B., 2007. Best practice guideline for the CFD simulation of flows in the urban environment, COST Office, Brussels, ISBN 3-00-018312-4.

Gousseau, P., Blocken, B., Stathopoulos, T., van Heijst, G.J.F., 2011a. CFD simulation of near-field pollutant dispersion on a high-resolution grid: a case study by LES and RANS for a building group in downtown Montreal. Atmospheric Environment 45, 428-438.

Gousseau, P., Blocken, B., van Heijst, G.J.F., 2011b. CFD simulation of pollutant dispersion around isolated buildings: On the role of convective and turbulent mass fluxes in the prediction accuracy. Journal of Hazardous Materials 194, 422-434.

Gousseau, P., Blocken, B., van Heijst, G.J.F., 2012. Large-eddy simulation of pollutant dispersion around a cubical building: Analysis of the turbulent mass transport mechanism by unsteady concentration and velocity statistics. Environmental Pollution 167, 47-57.

Green, S.R., 1992. Modelling turbulent air flow in a stand of widely-spaced trees. PHOENICS Journal of Computational Fluid Dynamics and Its Applications 23, 294-312.

Gromke, C., Ruck, B., 2007. Influence of trees on the dispersion of pollutants in an urban street canyon: Experimental investigation of the flow and concentration field. Atmospheric Environment 41, 3287-3302.

Gromke, C., Buccolieri, R., Sabatino, S.D., Ruck, B., 2008. Dispersion modeling in a street canyon with tree planting - Evaluation of CFD simulations with wind tunnel data. Atmospheric Environment 42, 8640-8650.

Gromke, C., Ruck, B., 2009. On the impact of trees on dispersion processes of traffic emissions in street canyons. Boundary-Layer Meteorology 131, 19-34.

Gu, Z.L., Jiao, J.Y., Su, J.W., 2011. Large-eddy simulation of the wind field and plume dispersion within different obstacle arrays using a dynamic mixing length subgrid-scale model. Boundary-Layer Meteorology 139, 439-455.

Hajra, B., Stathopoulos, T., Bahloul, A., 2010. Assessment of pollutant dispersion from rooftop stacks: ASHRAE, ADMS and wind tunnel simulation. Building and Environment 45, 2768-2777.

Hajra, B., Stathopoulos, T., Bahloul, A., 2011. The effect of upstream buildings on near-field pollutant dispersion in the built environment. Atmospheric Environment 45, 4930-4940.

Hall, R.C. (ed.), 1977. Evaluation of Modelling Uncertainty, Final report on contract EV5V- CT94-0531 for European Commission, March 1997, W.S. Atkins Report Ref. WSA/AM5017/R7.

Hall, D.J., Spanton, A.M., Bennett, M., Dunkerley, F., Griffiths, R.F., Fisher, B.E.A., Timmis, R.J., 2002. Evaluation of new generation atmospheric dispersion models. International Journal of Environment and Pollution 18, 22-32.

Hanna, S.R., Egan, B.A., et al., 2001. Evaluation of the ADMS, AERMOD, and ISC3 dispersion models with the OPTEX, Duke Forest, Kincaid, Indianapolis and Lovett field datasets. International Journal of Environment and Pollution 16, 301-314.

Hanna, S.R., Hansen, O.R., Dharmavaram, S., 2004. FLACS CFD air quality model performance evaluation with Kit Fox, MUST, Prairie Grass, and EMU observations. Atmospheric Environment 38, 4675-4687. 
Hanna, S.R., Brown, M.J., Camelli, F.E., Chan, S.T., Coirier, W.J., Hansen, O.R., Huber, A.H., Kim, S., Reynolds, R.M., 2006. Detailed simulations of atmospheric flow and dispersion in downtown Manhattan: An application of five computational fluid dynamics models. Bulletin of the American Meteorological Society 87, 1713-1726.

Hargreaves, D., Wright, N., 2007. On the use of the k- $-\varepsilon$ model in commercial CFD software tomodel the neutral atmospheric boundary layer. Journal of Wind Engineering and Industrial Aerodynamics 95, 355-369.

Harms, F., Leitl, B., Schatzmann, M., 2005. Comparison of tracer dispersion through a model of an idealized urban area from field (MUST) and wind tunnel measurements. Proceedings International Workshop on Physical Modelling of Flow and Dispersion Phenomena, London, Canada.

Harms, F., Leitl, B., Schatzmann, M., Patnaik, G., 2011. Validating LES-based flow and dispersion models. Journal of Wind Engineering and Industrial Aerodynamics 99(4), 289-295.

Higson, H.L., Griffiths, R.F., Jones, C.D., Biltoft, C., 1995. Effect of atmospheric stability on concentration fluctuations and wake retention times for dispersion in the vicinity of an isolated building. Environmetrics 6 , 571-581.

Hiraoka, H., 1993. Modelling of turbulent flows within plant/urban canopies. Journal of Wind Engineering and Industrial Aerodynamics 46\&47, 173-182.

Hsieh, K.-J., Lien, F.-S., Yee, E., 2007. Numerical modeling of passive scalar dispersion in an urban canopy layer. Journal of Wind Engineering and Industrial Aerodynamics 95, 1611-1636.

Huber, A.H. Snyder, W.H., 1982. Wind tunnel investigation of the effects of a rectangular shaped building on dispersion of effluent from short adjacent stacks. Atmospheric Environment 16, 2837-2848.

Hunter, L.J., Johnson, G.T. Watson, L.D., 1992. An investigation of three-dimensional characteristics of flow regimes within the urban canyon. Atmospheric Environment 26B, 425-432.

Iaccarino, G., Ooi, A., Durbin, P.A., Behnia, M., 2003. Reynolds averaged simulation of unsteady separated flow. International Journal of Heat and Fluid Flow 24, 147-156

Jicha, M., Pospisil, J., Katolicky, J., 2000. Dispersion of pollutants in street canyon under traffic induced flow and turbulence. Environmental Monitoring and Assessment 65, 343-351.

Kastner-Klein, P., Leitl, B., Pascheke, F., Schatzmann, M., 2004. Wind-tunnel simulation of the JOINT Urban 2003 tracer experiment. Proceedings of the AMS Symposium on Planning, Nowcasting, and Forecasting in the Urban Zone, January 11-15, Seattle, Washington, USA.

Katul, G. G., Mahrt, L., Poggi, D., Sanz, C., 2004. One- and two-equation models for canopy turbulence. Boundary-Layer Meteorology 113, 81-109.

Kato, M. \& Launder, B. E. 1993. The modeling of turbulent flow around stationary and vibrating square cylinders. Ninth Symposium on Turbulent Shear Flows, Paper 10-4, Kyoto, Japan.

Kim, J.J., Baik, J.J., 2003. Effects of inflow turbulence intensity on flow and pollutant dispersion in an urban street canyon. Journal of Wind Engineering and Industrial Aerodynamics 91, 309-329.

Kim, J.J., Baik, J.J., 2004. A numerical study of the effects of ambient wind direction on flow and dispersion in urban street canyons using the RNG $\kappa-\varepsilon$ turbulence model. Atmospheric Environment 38, 3039-3048.

Koeltzsch, K., 2000. The height dependence of the turbulent Schmidt number within the boundary layer. Atmospheric Environment 34, 1147-1151.

Kondo, H., Asahi, K., Tomizuka, T., Suzuki, M., 2006. Numerical analysis of diffusion around a suspended expressway by a multi-scale CFD model. Atmospheric Environment 40, 2852-2859.

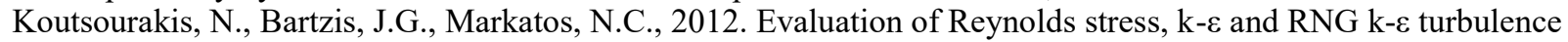
models in street canyon flows using various experimental datasets. Environmental Fluid Mechanics, DOI: $10.1007 / \mathrm{s} 10652-012-9240-9$

Kwak, K.-H., Baik, J.J., 2012. A CFD modeling study of the impacts of NOx and VOC emissions on reactive pollutant dispersion in and above a street canyon. Atmospheric Environment 46, 71-80.

Lateb, M., Masson, C., Stathopoulos, T., Bédard, C., 2010. Numerical simulation of pollutant dispersion around a building complex. Building and Environment 45, 1788-1798.

Launder, B.E., Spalding, D.B., 1972. Mathematical models of turbulence, Academic Press, New York.

Launder, B.E., Reece, G.J., Rodi, W., 1975. Progress in the development of a Reynolds-stress turbulence closure. Journal of Fluid Mechanics 68, 537-566.

Launder, B.E., 1978. Heat and mass transport. In Turbulence, 2nd Ed., P. Bradshaw, ed., Springer- Verlag, Berlin.

Leitl, B.M., Kastner-Klein, P., Rau, M., Meroney, R.N., 1997. Concentration and flow distributions in the vicinity of U-shaped buildings: Wind-tunnel and computational data. Journal of Wind Engineering and Industrial Aerodynamics $67 \& 68,745-755$.

Leitl, B.M., Meroney, R., 1997. Car exhaust dispersion in a street canyon. Numerical critique of a wind tunnel experiment. Journal of Wind Engineering and Industrial Aerodynamics 67 \& 68, 293-304. 
Li, W., Meroney, R.N., 1983a. Gas dispersion near a cubical model building, Part I, Mean concentration measurements. Journal of Wind Engineering and Industrial Aerodynamics 12, 15-33.

Li, W.W., Meroney, R.N., 1983b. Gas dispersion near a cubical model building, Part II, Concentration fluctuation measurements. Journal of Wind Engineering and Industrial Aerodynamics 12, 35-47.

Li, X.X., Liu, C.H., Leung, D.Y.C., Lam, K.M., 2006. Recent progress in CFD modelling of wind field and pollutant transport in street canyons. Atmospheric Environment 40, 5640-5658.

Li, X.X., Liu, C.H., Leung, D.Y.C., 2008. Large-eddy simulation of flow and pollutant dispersion in high-aspect-ratio urban street canyons with wall model. Boundary-Layer Meteorology 129, 249-268.

Li, X.-X., Liu, C.-H., Leung, D.Y.C., 2009. Numerical investigation of pollutant transport characteristics inside deep urban street canyons. Atmospheric Environment 43, 2410-2418.

Li, Y., Stathopoulos, T., 1997. Numerical evaluation of wind-induced dispersion of pollutants around a building. Journal of Wind Engineering and Industrial Aerodynamics 67-68, 757-766.

Li, Y, Stathopoulos, T., 1998. Computational evaluation of pollutant dispersion around buildings: estimation of numerical errors. Journal of Wind Engineering and Industrial Aerodynamics 77-78, 619-630.

Lien, F.S., Yee, E., Ji, H., Hsieh, K.J., 2008. Partially resolved numerical simulation and RANS modeling of flow and passive scalar transport in an urban environment. Journal of Wind Engineering and Industrial Aerodynamics 96, 1832-1842.

Liu, C.H., Barth, M.C., 2002. Large-Eddy Simulation of Flow and Scalar Transport in a Modeled Street Canyon. Journal of the Applied Meteorology 41, 660-673.

Liu, C., Leung. D., Barth, M., 2005. On the prediction of air and pollutant exchange rates in street canyons of different aspect ratios using large-eddy simulation. Atmospheric Environment 39, 1567-1574.

Liu, J., Chen, J.M., Black, T.A., Novak, M.D., 1996. E- $\varepsilon$ modelling of turbulent airflow downwind of a model forest edge. Boundary-Layer Meteorology 77, 21-44.

Liu, Y.S., Cui, G.X., Wang, Z.S., Zhang, Z.S., 2011. Large eddy simulation of wind field and pollutant dispersion in downtown Macao. Atmospheric Environment 45, 2849-2859.

Lyn, D.A., Eina, S., Rodi, V,W., Park, J.-H., 1995. A laser-Doppler velocimetry study of ensemble-averaged characteristics of the turbulent near wake of a square cylinder. Journal of Fluid Mechanics 304, 285-319.

Macdonald, R., Griffiths, R.F., Hall, D.J., 1998. A comparison of results from scaled field and wind tunnel modelling of dispersion in arrays of obstacles. Atmospheric Environment 32, 3845-3862.

Macdonald, R., 2003. Theory and objectives of air dispersion modelling. Modelling Air Emissions for Compliance MME 474A Wind Engineering, December 2003.

Martilli, A., Santiago, J.L., 2007. CFD simulation of airflow over a regular array of cubes. Part II: analysis of spatial average properties. Boundary-Layer Meteorology 122, 635-654.

Mavroidis, I., Griffiths, R.F., Hall, D.J., 2003. Field and wind tunnel investigations of plume dispersion around single surface obstacles. Atmospheric Environment 37, 2903-2918.

Mavroidis, I., Andronopoulos, S., Bartzis, J.G., Griffiths, R.F., 2007. Atmospheric dispersion in the presence of a three-dimensional cubical obstacle: Modelling of mean concentration and concentration fluctuations. Atmospheric Environment 41, 2740-2756.

Mavroidis, I., Andronopoulos, S., Bartzis, J.G., 2012. Computational simulation of the residence of air pollutants in the wake of a 3-dimensional cubical building. The effect of atmospheric stability. Atmospheric Environment 63, 189-202.

Meroney, R.N., 1990. Bluff-body aerodynamics influence on transport and diffusion. Journal of Wind Engineering and Industrial Aerodynamics 33, 21-33.

Meroney, R.N., Pavageau, M., Rafailidis, S., Schatzmann, M., 1996. Study of line source characteristics for 2-D physical modelling of pollutant dispersion in street canyons. Journal of Wind Engineering and Industrial Aerodynamics 62, 37-56.

Meroney, R.N., Leitl, B.M., Rafailidis, S., Schatzmann, M., 1999. Wind tunnel and numerical modeling of flow and dispersion about several building shapes. Journal of Wind Engineering and Industrial Aerodynamics 81, 333-345.

Milliez, M., Carissimo, B., 2007. Numerical simulations of pollutant dispersion in an idealized urban area, for different meteorological conditions. Boundary-Layer Meteorology 122, 321-342.

Milliez, M., Carissimo, B., 2008. Computational fluid dynamical modelling of concentration fluctuations in an idealized urban area. Boundary-layer Meteorology 127, 241-259.

Mochida, A., Lun, I.Y.F., 2008. Prediction of wind environment and thermal comfort at pedestrian level in urban area. Journal of Wind Engineering and Industrial Aerodynamics 96, 1498-1527. 
Mochida, A., Tabata, Y., Iwata, T., Yoshino, H., 2008. Examining tree canopy models for CFD prediction of wind environment at pedestrian level. Journal of Wind Engineering and Industrial Aerodynamics 96, 1667-1677.

Mochida, A., Iizuka, S., Tominaga, Y., Lun, I.Y.-F., 2011. Up-scaling CWE models to include mesoscale meteorological influences. Journal of Wind Engineering and Industrial Aerodynamics 99, 187-198.

Mokhtarzadeh-Dehghan, M.R., Akcayoglu, A., Robins, A.G., 2012. Numerical study and comparison with experiment of dispersion of a heavier-than-air gas in a simulated neutral atmospheric boundary layer. Journal of Wind Engineering and Industrial Aerodynamics 110, 10-24.

Moon, D., Albergel, A., Jasmin, F., Thibaut, G., 1997. The use of the MERCURE CFD code to deal with an air pollution problem due to building wake effects. Journal of Wind Engineering and Industrial Aerodynamics 67-68, 781-791.

Moonen, P., Dorer, V., Carmeliet, J., 2012. Effect of flow unsteadiness on the mean wind flow pattern in an idealized urban environment. Journal of Wind Engineering and Industrial Aerodynamics 104-106, 389-396.

Murakami, S., Mochida, A., Hayashi, Y., Hibi, K., 1990. Numerical simulation of velocity field and diffusion field in an urban area. Energy and Buildings 15-16, 345-356.

Murakami, S., 1993. Comparison of various turbulence models applied to a bluff body. Journal of Wind Engineering and Industrial Aerodynamics 46-47, 21-36.

Murakami, S., Mochida, A., 1995. On turbulent vortex shedding flow past 2D square cylinder predicted by CFD. Journal of Wind Engineering and Industrial Aerodynamics 54-55, 191-211.

Murakami, S., Mochida, A., Ooka, R., Kato, S., Iizuka, S., 1996. Numerical prediction of flow around a building with various turbulence models: Comparison of k- $\varepsilon$ EVM, ASM, DSM and LES with wind tunnel tests. ASHRAE Transactions 102, 741-753.

Murena, F., Favale, G., Vardoulakis, S., Solazzo, E., 2009. Modelling dispersion of traffic pollution in a deep street canyon: Application of CFD and operational models. Atmospheric Environment 43, 2303-2311.

Nazridoust, K., Ahmadi, G., 2006. Airflow and pollutant transport in street canyons. Journal of Wind Engineering and Industrial Aerodynamics 94, 491-522.

Neofytou, P., Haakana, M., Venetsanos, A., Kousa, A., Bartzis, J., Kukkonen, J., 2008. Computational fluid dynamics modelling of the pollution dispersion and comparison with measurements in a street canyon in Helsinki, Environmental Monitoring and Assessment 13, 439-448.

Ogawa, Y., Oikawa, S., Uehara, K., 1983. Field and wind tunnel study of the flow and diffusion around a model cube- II. Nearfield and cube surface flow and concentration patterns. Atmospheric Environment 17, 1161-1171.

Olesen, H.R., Baklanov, A., Bartzis, J., Barmpas, F., Berkowicz, R., Brzozowski, K., Buccolieri, R., Carissimo, B., Costa, A., Di Sabatino, S., Efthimiou, G., Franke, J., Goricsan, I., Hellsten, A., Ketzel, M., Leitl, B., Nuterman, R., Polreich, E. Tavares, R., 2008. The MUST model evaluation exercise: Patterns in model performance. Proceedings 12th International Conference on Harmonization within Atmospheric Dispersion Modelling for Regulatory Purposes, Cavtat, Croatia, 403-408.

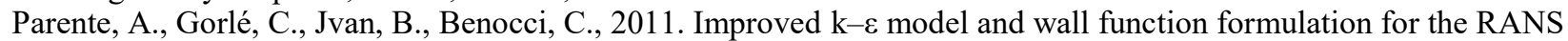
simulation of ABL flows. Journal of Wind Engineering and Industrial Aerodynamics 99, 267-278.

Pasquill, F. and Smith, F.B., 1983. Atmospheric diffusion. 3rd ed. Chichester, England: Ellis Horwood Ltd.

Patnaik, G., Boris, J.P., Young, T.R., 2007. Large scale urban contaminant transport simulations with Miles. Journal of Fluids Engineering 129, 1524-1532.

Pontiggia, M., Derudi, M., Alba, M., Scaioni, M., Rota, R., 2010. Hazardous gas releases in urban areas: assessment of consequences through CFD modelling. Journal of Hazardous Materials 176, 589-596.

Pontiggia, M., Landucci, G., Busini, V., Derudi, M., Alba, M., Scaioni, M., Bonvicini, S., Cozzani, V., Rota, R., 2011. CFD model simulation of LPG dispersion in urban areas. Atmospheric Environment 45, 3913-3923.

Riddle, A., Carruthers, D., Sharpe, A., McHugh, C., Stocker, J., 2004. Comparisons between FLUENT and ADMS for atmospheric dispersion modelling. Atmospheric Environment 38, 1029-1038.

Robins, A.G., 1979. The development and structure of simulated neutrally stable atmospheric boundary layers. Journal of Wind Engineering and Industrial Aerodynamics 4, 71-100.

Robins, A., 2003. Wind tunnel dispersion modelling some recent and not so recent achievements. Journal of Wind Engineering and Industrial Aerodynamics 91, 1777-1790.

Robins, A., Carruthers, D., McHugh, C., 1997. The ADMS building effects module. International Journal of Environment and Pollution 8, 708-717.

Robins, A.G., Castro, I. P., 1977a. A wind tunnel investigation of plume dispersion in the vicinity of a surface mounted cube - I. The flow field. Atmospheric Environment 11, 291-297.

Robins, A.G., Castro, I. P., 1977b. A wind tunnel investigation of plume dispersion in the vicinity of a surface mounted cube - II. The concentration field. Atmospheric Environment 11, 299-311. 
Robins, A., Castro, I., Hayden, P., Steggel, N., Contini, D., Heist, D., John Taylor, T., 2001. A wind tunnel study of dense gas dispersion in a stable boundary layer over a rough surface. Atmospheric Environment 35, 2253-2263.

Robins, A.G., Hall, R., Cowan, I.R., Bartzis, J.G., Albergel, A., 2000. Evaluating modelling uncertainty in CFD predictions of building affected dispersion. International Journal of Environment and Pollution 14, 52-64.

Robins, A., MacDonald, R., 2001. Review of flow and dispersion in the vicinity of groups of buildings, Annexure B, University of Surrey.

Rodi, W., 1997. Comparison of LES and RANS calculations of the flow around bluff bodies. Journal of Wind Engineering and Industrial Aerodynamics 69-71, 55-75.

Rogers, M.M., Mansour, N.N., Reynolds, W.C., 1989. An algebraic model for the turbulent flux of a passive scalar. Journal of Fluid Mechanics 203, 77-101.

Rossi, R., Iaccarino, G., 2009. Numerical simulation of scalar dispersion downstream of a square obstacle using gradient-transport type models. Atmospheric Environment 43, 2518-2531.

Rossi, D., Philips, D., Iaccarino, G., 2010. A numerical study of scalar dispersion downstream of a wall-mounted cube using direct simulations and algebraic flux models. International Journal of Heat and Fluid Flow 31, 805-819.

Sada, K., Sato, A., 2002. Numerical calculation of flow and stack-gas concentration fluctuation around a cubical building. Atmospheric Environment 36, 5527-5534.

Salim S.M., Buccolieri, R., Chan, A., Di Sabatino, S., 2011. Numerical simulations of atmospheric pollutant dispersion in an urban street canyon: Comparison between RANS and LES. Journal of Wind Engineering and Industrial Aerodynamics 99, 103-113.

Santiago, J.L., Dejoan, A., Martilli, A., Martin, F, Pinelli, A., 2010. Comparison between large-eddy simulation and Reynolds-averaged Navier-Stokes computations for the MUST field experiment. Part I: Study of the Flow for an Incident Wind Directed Perpendicularly to the Front Array of Containers. Boundary-Layer Meteorology 135, 109-132.

Santiago, J.L., Martilli, A., Martín, F., 2007. CFD simulation of airflow over a regular array of cubes. Part I: Three-dimensional simulation of the flow and validation with wind-tunnel measurements. Boundary-Layer Meteorology 122, 609-634.

Santos, J.M., Griffiths, R.F., Roberts, I.D., Reis Jr., N.C., 2005. A field experiment on turbulent concentration fluctuations of an atmospheric tracer gas in the vicinity of a complex-shaped building. Atmospheric Environment 39, 4999-5012.

Santos, J.M., Reis, N.C., Goulart, E.V., Mavroidis, I., 2009. Numerical simulation of flow and dispersion around an isolated cubical building: the effect of the atmospheric stratification. Atmospheric Environment 43, 5484-5492.

Sanz, C., 2003. A note on k- $\varepsilon$ modelling of vegetation canopy air-flows. Boundary-Layer Meteorology 108, 191-197.

Schatzmann, M., Rafailidis, S., Pavageau, M., 1997. Some remarks on the validation of small-scale dispersion models with field and laboratory data. Journal of Wind Engineering and Industrial Aerodynamics 67\&68, 885-893.

Schatzmann, M., Leitl, B., 2002. Validation and application of obstacle-resolvingurban dispersion models. Atmospheric Environment 36, 4811-4821.

Schatzmann, M., Leitl, B, 2011. Issues with validation of urban flow and dispersion CFD models. Journal of Wind Engineering and Industrial Aerodynamics 99, 169-186.

Schatzmann, M., Olesen, H., Franke, J. (Eds.), 2009. COST 732 Model Evaluation Case Studies: Approach and Results, COST Office, Brussels, Belgium.

Selvam, R.P., 1997b. Numerical simulation of pollutant dispersion around a building using FEM. Journal of Wind Engineering and Industrial Aerodynamics 67-68, 805-814.

Shao, J., Liu, J., Zhao, J., 2012. Evaluation of various non-linear k- $\varepsilon$ models for predicting wind flow around an isolated high-rise building within the surface boundary layer. Building and Environment 57, 145-155.

Shi, R.F., G.X. Cui, Z.S. Wang, C.X. Xu, Z.S. Zhang, 2008. Large eddy simulation of wind field and plume dispersion in building array. Atmospheric Environment 42, 1083-1097.

Shimada, K., Ishihara, T., 2002. Application of a modified k- $\varepsilon$ model to the prediction of aerodynamic characteristics of rectangular cross-section cylinders, Journal of Fluids and Structures 16, 465-485.

Shiono, K., Scott, C.F., Kearney, D., 2003. Predictions of solute transport in a compound channel using turbulence models, Journal of Hydraulic Research 41, 247-258.

Simoes, F. J. M., Wang, S. S. Y., 1997. Numerical prediction of three dimensional mixing in a compound channel. Journal of Hydraulic Research 5, 619-642.

Sini, J.-F., Anquetin, S., Mestayer, P.G., 1996. Pollutant dispersion and thermal effects in urban street canyons. Atmospheric Environment 30, 2659-2677. 
Smith, W.S, Reisner, J.M., Kao, C.-Y.J, 2001. Simulations of flow around a cubical building: comparison with towing-tank data and assessment of radiatively induced thermal effects. Atmospheric Environment 35, 3811-3821.

Solazzo, E; Cai, XM; Vardoulakis, S., 2009. Improved parameterisation for the numerical modelling of air pollution within an urban street canyon. Environmental Modelling \& Software 24, 381-388.

Stathopoulos, T., 2002. The Numerical Wind Tunnel for Industrial Aerodynamics: Real or Virtual in the New Millennium?. Wind and Structures 5, 193-208.

Stathopoulos, T., Lazure, L., Saathoff, P., Gupta, A., 2004. The effect of stack height, stack location and rooftop structures on air intake contamination: a laboratory and full-scale study. Report R-392, IRSST, Quebec.

Stern, A.C., 1976. Air Pollution: Volume I - Air Pollutants, Their Transformation and Transport, Academic Press, New York.

Svensson U., Häggkvist, K., 1990. A two-equation turbulence model for canopy flows. Journal of Wind Engineering and Industrial Aerodynamics 35, 201-211.

Tabor, G.R., Baba-Ahmadi, M.H., 2010. Inlet conditions for large eddy simulation: a review. Computers \& Fluids 39, $553-567$.

Tominaga, Y., Murakami, S., Mochida, A., 1997. CFD prediction of gaseous diffusion around a cubic model using a dynamic mixed SGS model based on composite grid technique. Journal of Wind Engineering and Industrial Aerodynamics 67-68, 827-841.

Tominaga, Y., Stathopoulos, T., 2007. Turbulent Schmidt numbers for CFD analysis with various types of flowfield. Atmospheric Environment 41, 8091-8099.

Tominaga, Y., Mochida, A., Murakami, S., Sawaki, S., 2008a. Comparison of various revised k- $\varepsilon$ models and LES applied to flow around a high-rise building model with 1:1:2 shape placed within the surface boundary layer. Journal of Wind Engineering and Industrial Aerodynamics 96, 389-411.

Tominaga, Y., Mochida, A., Yoshie, R., Kataoka, H., Nozu, T., Yoshikawa, M., Shirasawa, T., 2008b. AIJ guidelines for practical applications of CFD to pedestrian wind environment around buildings. Journal of Wind Engineering and Industrial Aerodynamics 96, 1749-1761.

Tominaga, Y., Stathopoulos, T., 2009. Numerical simulation of dispersion around an isolated cubic building : Comparison of various types of k- $\varepsilon$ models. Atmospheric Environment 43, 3200-3210.

Tominaga, Y., Stathopoulos, T., 2010. Numerical simulation of dispersion around an isolated cubic building: Model evaluation of RANS and LES. Building and Environment 45, 2231-2239.

Tominaga, Y., Stathopoulos, T., 2011. CFD modeling of pollution dispersion in a street canyon: Comparison between LES and RANS. Journal of Wind Engineering \& Industrial Aerodynamics 99, 340-348.

Tominaga, Y., Stathopoulos, T., 2012. CFD modeling of pollution dispersion in building array: Evaluation of turbulent scalar flux modeling in RANS model using LES results. Journal of Wind Engineering \& Industrial Aerodynamics 104-106, 484-491.

Tseng, Y.H., Meneveau, C., Parlange, M.B., 2006. Modeling flow around bluff bodies and predicting urban dispersion using large eddy simulation. Environmental Science \& Technology 40, 2653-2662.

Uehara, K., Murakami, S., Oikawa, S., Wakamatsu, S., 2000. Wind tunnel experiments on how thermal stratification affects flow in and above urban street canyons. Atmospheric Environment 34, 1553-1562.

Vardoulakis, S., Fisher, B.E., Pericleous, K., Gonzalez-Flesca, N., 2003. Modelling air quality in street canyons: a review. Atmospheric Environment 37, 155-182.

Venetsanos, A. G., Vlachogiannis, D., Papadopoulos, A., Bartzis, J. G., Andronopoulos S., 2002. Studies on Pollutant Dispersion from Moving Vehicles. Water, Air and Soil Pollution 2, 325-337.

VDI, 2005. Environmental meteorology - Prognostic microscale windfield models - Evaluation for flow around buildings and obstacles, VDI guideline 3783, Part 9, Beuth Verlag, Berlin.

Walton, A., Cheng, A.Y.S., Yeung, W.C., 2002. Large-eddy simulation of pollution dispersion in an urban street canyon - Part I: Comparison with field data. Atmospheric Environment 36, 3601-3613.

Walton, A., Cheng, A.Y.S., 2002. Large-eddy simulation of pollution dispersion in an urban street canyon - Part II: Idealised canyon simulation. Atmospheric Environment 36, 3615-3627.

Wang, B.-C., Yee, E., Lien, F.S., 2009. Numerical study of dispersing pollutant clouds in a built-up environment. International Journal of Heat and Fluid Flow 30, 3-19.

Wang, X., McNamara, K.F., 2006. Evaluation of CFD Simulation using RANS Turbulence Models for Building Effects on Pollutant Dispersion. Environmental Fluid Mechanics 6, 181-202.

Wilson, D.J. 1982. Critical wind speeds for maximum exhaust gas reentry from flush vents at roof level intakes. ASHRAE Transactions 88, 503-513. 
Wilson, D. J. 1983. A design procedure for estimating air intake contamination from nearby exhaust vents, ASHRAE Transactions 89, 136-152.

Wilson, D.J., Lamb, B., 1994. Dispersion of exhaust gases from roof level stacks and vents on a laboratory building. Atmospheric Environment 28, 3099-3111.

Wood, C. R., Barlow, J. F., Belcher, S.E., Dobre, A., Arnold, S.J., Balogun, A.A., Lingard, J. J. N., Smalley, R. J., Tate, J. E., Tomlin, A. S., Britter, R. E., Cheng, H., Martin, D., Petersson, F. K., Shallcross, D. E., White, I. R., Neophytou, M. K., Robins, A.G. 2009. Dispersion experiments in central London: The 2007 DAPPLE Project. Bulletin of the American Meteorological Society 90, 955-969.

Xie, X. , Huang, Z., Wang, J., Xie, Z., 2005. The impact of solar radiation and street layout on pollutant dispersion in street canyon. Building and Environment 40, 201-212.

Xie, Z.-T., Castro, I.P., 2008. Efficient generation of inflow conditions for large eddy simulation of street-scale flows. Flow Turbulence Combustion 81, 449-470.

Xie, Z.-T., Castro, I.P., 2009. Large-eddy simulation for flow and dispersion in urban streets. Atmospheric Environment 43, 2174-2185.

Xie, Z.-T., 2010. Large-eddy simulation of stratification effects on dispersion in urban environments. Journal of Hydrodynamics, Ser. B 22, Supplement 1, 1003-1008.

Xie, Z.-T., 2011. Modelling of street-scale flows and dispersion in realistic winds -towards coupling with mesoscale meteorological models. Boundary-Layer Meteorology 141, 53-75.

Xie, Z.-T., Hayden, P., Wood, C. R., 2013. Large-eddy simulation of approaching-flow stratification on dispersion over arrays of buildings. Atmospheric Environment 71, 64-74.

Yakhot, A., Liu, H.P., Nikitin, N. 2006. Turbulent flow around a wall-mounted cube: a direct numerical simulation. International Journal of Heat and Fluid Flow 27, 994-1009.

Yakhot, V., Orszag, S.A., Thangam, S., Gatski, T.B., Speziale, C.G., 1992. Development of turbulence models for shear flows by a double expansion technique. Physics of Fluids A4, 1510-1520.

Yang, Y., Shao, Y., 2008. Numerical simulations of flow and pollution dispersion in urban atmospheric boundary layers. Environmental Modelling \& Software 23, 906-921.

Yang, Y., Gu, M., Chen, S., Jin, X., Jin, H., Gu, M., Chen, S., 2009. New inflow boundary conditions for modeling the neutral equilibrium atmospheric boundary layer in computational wind engineering. Journal of Wind Engineering and Industrial Aerodynamics 97, 88-95.

Yassin, M. F., 2013. A wind tunnel study on the effect of thermal stability on flow and dispersion of rooftop stack emissions in the near wake of a building, Atmospheric Environment 65, 89-100.

Yee, E., Biltoft C.A., 2004. Concentration fluctuation measurements in a plume dispersing through a regular array of obstacles. Boundary-Layer Meteorology 111, 363-415.

Yee, E., Gailis R.M., Hill, A., Hilderman, T., Kiel, D., 2006, Comparison of wind-tunnel and water-channel simulations of plume dispersion through a large array of obstacles with a scaled field experiment. Boundary-Layer Meteorology 121, 389-432.

Yee, E., Wang, B.-C., Lien, F.-S., 2009. Probabilistic Model for Concentration Fluctuations in Compact-Source Plumes in an Urban Environment. Boundary-Layer Meteorology 130, 169-208.

Yoshie, R., Jiang, G., Shirasawa, T., Chung, J., 2011. CFD simulations of gas dispersion around high-rise building in non-isothermal boundary layer. Journal of Wind Engineering and Industrial Aerodynamics 99, 279-288.

Younis, B.A., Speziale, C.G., Clark, T.T., 2005. A rationale model for the turbulent scalar fluxes. Proceedings of The Royal Society A 461, 575-594.

Younis B. A., Zhou. Y., 2006. Accounting for Mean-Flow Periodicity in Turbulence Closures. Physics of Fluids 18, 018102 .

Zhang, Y.Q., Huber, A.H., Arya, S.P., Snyder, W.H., 1993. Numerical Simulation to determine the effects of incident wind shear and turbulence on the flow around a building. Journal of Wind Engineering and Industrial Aerodynamics 46\&47, 129-134.

Zhang, Y.Q., Arya, S.P., Snyder, W.H., 1996. A comparison of numerical and physical modeling of stable atmospheric flow and dispersion around a cubical building. Atmospheric Environment 30, 1327-1345.

Zhang, Y.-W., Gu, Z.-L., Cheng, Y., Lee, S.-C., 2011. Effect of real-time boundary wind conditions on the air flow and pollutant dispersion in an urban street canyon - Large eddy simulations. Atmospheric Environment 45, 3352-3359.

Zhang, Z.S., Cui, G.X., Shi, R.F., Wang. Z.S., Xu, C.X., 2009. Large Eddy Simulation of traffic pollutant dispersion in a residence area, Turbulence, Heat and Mass Transfer 6, in: K. Hanjalić, Y. Nagano and S. Jakirlić (Eds.), (C) 2009 Begell House, Inc. 